\title{
Democratic Mass Matrices From Five Dimensions
}

\author{
A. Soddu* and N-K. Trant \\ Dept. of Physics, University of Virginia, \\ 382 McCormick Road, P. O. Box 400714, \\ Charlottesville, Virginia 22904-4714
}

(Dated: December 12, 2018)

\begin{abstract}
We reconstruct the Standard Model (SM) quark masses and the Cabibbo-Kobayashi-Maskawa (CKM) matrix from a five-dimensional model, with the fifth dimension compactified on an $S^{1} / Z_{2}$ orbifold. Fermions are localized only at the orbifold fixed points and the induced quark mass matrices are almost democratic. Two specific versions of our model with 15 and 24 parameters are presented, and for both versions we can reproduce the quark mass spectrum and CKM matrix correctly to the level they are observed in current experiments.
\end{abstract}

PACS numbers: $11.25 . \mathrm{Mj}, 12.15 . \mathrm{Ff}$

${ }^{*}$ Electronic address: as7yf@galileo.phys.virginia.edu

${ }^{\dagger}$ Electronic address: nt6b@galileo.phys.virginia.edu 


\section{INTRODUCTION}

The Standard Model (SM) has been the most satisfactory and widely recognized theory of particle interaction. To an extent, this is an effective theory, whose certain parameters are estimated and then refined by increasing high-precision experiments. However, this also means that the dynamical origin of some of these parameters is not found within the SM. One example is the pattern of family mixing characterized by the Cabibbo-Kobayashi-Maskawa (CKM) matrix, which in turn is related to the SM fermion mass spectrum and CP violation.

Recently, considerable attention has been directed to phenomenological models with extra dimensions since among many other things, they can offer potential answers to puzzling questions related to the SM.

In theories with compact extra dimensions, each original field in higher dimensional space can be effectively "viewed" as a tower of Kaluza-Klein (KK) states in equivalent 4-d theories after compactification processes. If SM is assumed to be the low-energy manifestation of a higher dimensional theory, the tower's lowest state (or KK zero mode) is identified as a SM field. In [1] (see also earlier works [2, 3]), by introducing a $Z_{2}$-invariant Yukawa interaction between a background scalar field and a fermion field in 5 -d theory, after a $S^{1} / Z_{2}$ compactification, one can obtain a non-trivial (i.e. localized) solution for the KK zero-mode wavefunction along the fifth dimension (brane scenario). From a 4-d point of view, any interaction term is now associated with a coupling being the overlap integration of extra dimensional wavefunctions of related fields. One then can flexibly control this 4-d theory effective coupling by regulating the localized wavefunctions along the extra dimension. This very interesting mechanism has found potential application in many problems such as proton decay suppression [3], 4], fermion mass hierarchy [5, 6, 7, 8, 9], CP violation [10, 12], etc.

In this work, we discuss the problem of quark mass spectrum and mixing angles by making extensive use of a democratic structure for the mass matrices. We found that a democratic structure for the mass matrices (DMM) is a particularly convenient choice, because it raises quite naturally in brane picture, and it can adequately generate both quark mass spectrum and CKM matrix to the precision determined by current experimental data. Previous works dealing with fermion mass hierarchy and CP violation within splitfermion scenario [6, 9, 10, 11, 12] focus on placing fermion families on different positions in the bulk, with the possibility to make mass matrix elements, originating from couplings 
between geographically very distant families, approximately zero. These highly hierarchical mass matrix approach however requires additional techniques (see section II) because naively localizing fermions in an arbitrary position along the extra dimension may contradict $S^{1} / Z_{2}$ orbifold compactification being used. In the DMM approach, we can avoid this subtlety by localizing all fields only at the two fixed points of the orbifold, while we break the family symmetry by modifying the detailed shapes of their wavefuctions. Further, the approach turns out to be more symmetric too.

Our work is structured as follows: in section II we introduce the Lagrangian to generate pure-phase mass matrices (PPMM) and DMM within a fermion localization mechanism, in section III we give the description of parameter space, in section IV we present the numerical method and report the results for the mass spectrum and CKM matrix for the 24 parameter version of our model and in section $\mathrm{V}$ for the 15 parameter version. Finally we give a brief conclusion of the work in section VI.

\section{FORMALISM}

\section{A. Fermion Localization Mechanism}

We first briefly review the mechanism of fermion localization in extra dimension 1, 2,

3]. We begin with the 5-d Lagrangian for a single massless fermion interacting with a real background scalar field. The fifth dimension is compact with support $[0, L]$. The generalization to the case with different families of fermions is straightforward.

$$
\begin{aligned}
\mathcal{L}= & \bar{\psi}(x, y)\left(i \gamma^{\mu} \partial_{\mu}-\gamma^{5} \partial_{y}-f \phi(x, y)\right) \psi(x, y) \\
& +\frac{1}{2} \partial^{\mu} \phi(x, y) \partial_{\mu} \phi(x, y)-\frac{1}{2} \partial_{y} \phi(x, y) \partial_{y} \phi(x, y)-\frac{\lambda}{4}\left(\phi^{2}(x, y)-V^{2}\right)^{2} .
\end{aligned}
$$

It is important that this Lagrangian is invariant under a $Z_{2}$ symmetry [19]

$$
\begin{gathered}
\phi(x, y) \rightarrow \Phi(x, y) \equiv-\phi(x, L-y), \\
\psi(x, y) \rightarrow \Psi(x, y) \equiv \gamma_{5} \psi(x, L-y),
\end{gathered}
$$

To obtain the chiral zero mode for fermions as they are in the SM, it is necessary to compactify the 5-d theory on an $S^{1} / Z_{2}$ orbifold through the imposition of the following relations on the fields

$$
\phi(x,-y)=\Phi(x, L-y)=\phi(x, 2 L-y),
$$




$$
\psi(x,-y)=\Psi(x, L-y)=\psi(x, 2 L-y),
$$

which constrain the fermion left-handed component and the scalar wavefunctions to be antisymmetric, and the fermion right-handed component to be symmetric at the orbifold fixed points $y=0, L$. This in turn gives rise to a stable and non-constant VEV solution for the background scalar field when $L$ is sufficiently large $\left(L>\sqrt{\frac{1}{\lambda V^{2}}}\right)$

$$
<\phi(x, y)>=h(y)=V \tanh \left(\mu \frac{y}{L}\right) \tanh \left(\mu\left(1-\frac{y}{L}\right)\right)+\mathcal{O}\left(e^{-\mu}\right),
$$

where $\mu \equiv L \sqrt{\frac{\lambda V^{2}}{2}}$ characterizes the extent of the brane in the transverse direction, to which Standard Model chiral quarks are going to be confined. Clearly, VEV kink-antikink approximation (6) holds only for $\mu>1$. After performing a chiral decomposition

$$
\psi(x, y)=\psi_{R}(x) \xi_{R}(y)+\psi_{L}(x) \xi_{L}(y)
$$

one obtains the massless (zero-mode) fermion wavefunctions satisfying the motion equation associated with Lagrangian (1)

$$
\begin{aligned}
& \xi_{R}(y)=\frac{1}{N_{R}} \exp \left(-f \int_{0}^{y} h\left(y^{\prime}\right) d y^{\prime}\right)=\frac{1}{N_{R}} \exp \left[F\left(\mu y+\frac{1}{\tanh \mu} \ln \frac{\cosh \mu(1-y)}{\cosh \mu y \cosh \mu}\right)\right], \\
& \xi_{L}(y)=\frac{1}{N_{L}} \exp \left(f \int_{0}^{y} h\left(y^{\prime}\right) d y^{\prime}\right)=\frac{1}{N_{L}} \exp \left[-F\left(\mu y+\frac{1}{\tanh \mu} \ln \frac{\cosh \mu(1-y)}{\cosh \mu y \cosh \mu}\right)\right],
\end{aligned}
$$

where $F \equiv f \sqrt{\frac{2}{\lambda}}$ and $N_{L, R}$ are the normalization factors.

Now let us mention some important properties of these zero-mode solutions which are relevant to the present work.

First, both solutions (8) and (9) are symmetric at the orbifold fixed points $y=0, L$, so the right component survives and the left component vanishes by contradiction with the orbifold boundary conditions (5) assuring the single-handedness of SM fermions. By inverting the sign of $\gamma_{5}$ in (3) one can change the chirality of the surviving fermion.

Second, the sign of the Yukawa coupling $f$ decides the localization position of the surviving chiral zero-mode fermions along the extra dimension; if $f>0$ it is $y=0$, if $f<0$ it is $y=L$. Localizing fermions at an arbitrary location other than the two fixed points in the bulk requires additional extensions of the Lagrangian such as odd-mass terms [5] or two background scalar fields [6]. In the next section, sticking just to the minimal localization mechanism, we exhaust all possibilities of placing different $S U(2)_{W}$ representations $Q, U$ and $D$ at the two fixed points and find that, in all configurations, this is indeed sufficient to 
obtain the right quark mass spectrum and CKM matrix. Remarkably, this minimal localization mechanism also features a democratic structure for the quark mass matrices, because fields of identical $S U(2)_{W} \times U(1)_{Y}$ gauge symmetry $\left(Q_{i}\right.$ 's,$U_{i}$ 's or $D_{i}$ 's) are localized at the same point along the extra dimension. Small deviations from a democratic mass matrix, which is necessary in any realistic model, are realized in our approach by slightly modifying the fermions wavefunctions.

Third, in the difference with other works in literature, here we make use of the exact solution for the zero-mode wavefunctions (8), (9) in place of a Gaussian profile approximation.

\section{B. Quark Flavor Mixing}

In the spirit of SM, we now introduce three $S U(2)_{W}$ doublets $Q_{i}$ and six $S U(2)_{W}$ singlets $U_{i}, D_{i}(i=1,2,3)$ whose zero modes are identified respectively with the SM quark chiral components $q_{i}, u_{i}, d_{i}$ after orbifold compactification. The Higgs doublet zero mode is assumed to be uniform along transverse direction $(H(x, y)=H(x) / \sqrt{L})$.

We construct a general 5-d Lagrangian concerning three fermion families

$$
\begin{aligned}
\mathcal{L}_{f} & =\sum_{i=1}^{3} \bar{Q}_{i}(x, y) i \not{ }_{5} Q_{i}(x, y)+\left(Q_{i} \leftrightarrow U_{i}\right)+\left(Q_{i} \leftrightarrow D_{i}\right) \\
& +\sum_{i, j=1}^{3} \kappa_{5, i j}^{u} \bar{Q}_{i}(x, y) i \sigma_{2} H^{*}(x, y) U_{j}(x, y)+\sum_{i, j=1}^{3} \kappa_{5, i j}^{d} \bar{Q}_{i}(x, y) H(x, y) D_{j}(x, y)+\text { h.c. }
\end{aligned}
$$

In order to obtain a PPMM in 4-d effective theory, we use the following ansatz for 5-d Yukawa couplings

$$
\kappa_{5, i j}^{u}=\kappa_{5}^{u} \exp \left(i \theta_{i j}^{u}\right) \quad \kappa_{5, i j}^{d}=\kappa_{5}^{d} \exp \left(i \theta_{i j}^{d}\right),
$$

with $\kappa_{5, i j}^{u}$ and $\kappa_{5, i j}^{d}$ real positive. We note in particular in the above ansatz that complex higher-dimensional Yukawa couplings have universal absolute values $\kappa_{5}^{u}$, $\kappa_{5}^{d}$ for both up and down sector, and family symmetry is broken only in the phases. The difference between $\kappa_{5}^{u}$ and $\kappa_{5}^{d}$ to eventually give rise to the up-down quark mass hierarchy can also be accommodated conveniently in the brane picture with more extra dimensions (section IIC). The SM quarks obtain masses via spontaneous symmetry breaking with the Higgs developing a $\operatorname{VEV} H(x, y) \rightarrow(0, v / \sqrt{2 L})^{T}$ (note that SM chiral fields $q_{u i}$ and $q_{d i}$ have identical extra-dimensional wavefunctions because they originally come from the same doublet in 
higher-dimensional theory) [20]

$$
\begin{aligned}
\int d y \mathcal{L}_{f} & \rightarrow \sum_{i=1}^{3} \bar{q}_{u i}(x) i \not \partial q_{u i}(x)+\left(q_{u i} \leftrightarrow q_{d i}\right)+\left(q_{u i} \leftrightarrow u_{i}\right)+\left(q_{u i} \leftrightarrow d_{i}\right) \\
& +\sum_{i, j=1}^{3}\left[\bar{q}_{u i}(x) M_{i j}^{u} u_{j}(x)+\bar{q}_{d i}(x) M_{i j}^{d} d_{j}(x)\right]+\text { h.c. },
\end{aligned}
$$

where

$$
\begin{aligned}
M_{i j}^{u} & =\frac{v}{\sqrt{2}} g_{Y u} \exp \left(i \theta_{i j}^{u}\right) \int d y \xi_{q i}(y) \xi_{u j}(y), \\
M_{i j}^{d} & =\frac{v}{\sqrt{2}} g_{Y d} \exp \left(i \theta_{i j}^{d}\right) \int d y \xi_{q i}(y) \xi_{d j}(y),
\end{aligned}
$$

with real, dimensionless effective couplings $g_{Y u}=\kappa_{5}^{u} / \sqrt{L}$ and $g_{Y d}=\kappa_{5}^{d} / \sqrt{L}$. One has to notice that a pure-phase structure for the matrices $M^{u}$ and $M^{d}$ arises when the left-handed zero mode of $Q_{i}$ are localized at the same position along the extra dimension independently of the family index $i$ and the same happens for the right-handed zero mode of $U_{i}$ and $D_{i}$. Then as it is clear from Eqs. (13) and (14) the elements of each matrix differ only by a phase factor.

We first perform the usual transformation from gauge eigenbasis to mass eigenbasis

$$
\begin{aligned}
u_{i} & =U_{R i j}^{u} u_{j}^{\prime}, & d_{i} & =U_{R i j}^{d} d_{j}^{\prime}, \\
q_{L i}^{u} & =U_{L i j}^{u} q_{L j}^{\prime u}, & q_{L i}^{d} & =U_{L i j}^{d} q_{L j}^{\prime d},
\end{aligned}
$$

where $U_{L}^{u}, U_{L}^{d}$ diagonalize respectively the matrices $\left(M^{u} M^{u \dagger}\right),\left(M^{d} M^{d^{\dagger}}\right)$

$$
\begin{gathered}
\operatorname{diag}\left(\left|m_{u}\right|^{2},\left|m_{c}\right|^{2},\left|m_{t}\right|^{2}\right)=U_{L}^{u \dagger}\left(M^{u} M^{u \dagger}\right) U_{L}^{u}, \\
\operatorname{diag}\left(\left|m_{d}\right|^{2},\left|m_{s}\right|^{2},\left|m_{b}\right|^{2}\right)=U_{L}^{d \dagger}\left(M^{d} M^{d^{\dagger}}\right) U_{L}^{d},
\end{gathered}
$$

and whose product gives the CKM matrix

$$
V_{C K M}=U_{L}^{u \dagger} U_{L}^{d}
$$

The origin of $\mathrm{CP}$ violation in weak interaction is related to the phase appearing in the CKM matrix, which by virtue of above relations comes from the complexity of mass matrices (it is well-known that real mass matrices do not give rise to $\mathrm{CP}$ violation). The localization mechanism in 5-d theory clearly provides a direct control over the modulus of each mass matrix element, but it does nothing to their phases. That is, a priori $M^{u}$ and $M^{d}$ may 
possess in total 18 arbitrary phases $\theta_{i j}^{u}, \theta_{i j}^{d}$. [21] In the present work, stemming from the interest in model's simplicity, we just attribute four phases $\phi_{u 1}, \phi_{u 2}, \phi_{d 1}, \phi_{d 2}$ to elements of $M^{u}$ (say $M_{12}^{u}, M_{23}^{u}$ ) and $M^{d}$ (say $M_{12}^{d}, M_{23}^{d}$ ). One can note here that hermitian matrices $\left(M^{u} M^{u \dagger}\right)$ and $\left(M^{d} M^{d^{\dagger}}\right)$ have altogether six phases, but two of them are non-physical and can be eliminated by a simultaneous transformation involving a single diagonal phase matrix $K=\operatorname{diag}(1, \exp (i \alpha), \exp (i \beta))\lfloor 10]$

$$
\begin{aligned}
& M^{u} M^{u \dagger} \rightarrow K M^{u} M^{u \dagger} K^{\dagger}, \\
& M^{d} M^{d^{\dagger}} \rightarrow K M^{d} M^{d^{\dagger}} K^{\dagger} .
\end{aligned}
$$

Now the 4 physical phases left in $M^{u} M^{u \dagger}, M^{d} M^{d^{\dagger}}$ can be reproduced by the chosen configuration with four phases in $M^{u}, M^{d}$.

Let us next consider the magnitude of mass matrix elements, whose complete expressions are

$$
\begin{aligned}
M_{i j}^{u}= & \frac{v}{\sqrt{2}} g_{Y u} \exp \left(i \theta_{i j}^{u}\right) \int d y \xi_{q i}(y) \xi_{u j}(y)= \\
& \frac{\exp \left(i \theta_{i j}^{u}\right)}{N_{q i} N_{u j}} \int d y \exp \left[F_{q i}\left(\mu_{q i} y+\frac{\tanh \mu_{q i}}{\ln } \frac{\cosh \mu_{q i}(1-y)}{\cosh \mu_{q i} y \cosh \mu_{q i}}\right)\right. \\
& \left.+F_{u j}\left(\mu_{u j} y+\frac{1}{\tanh \mu_{u j}} \ln \frac{\cosh \mu_{u j}(1-y)}{\cosh \mu_{u j} y \cosh \mu_{u j}}\right)\right], \\
M_{i j}^{d}= & \frac{v}{\sqrt{2}} g_{Y d} \exp \left(i \theta_{i j}^{d}\right) \int d y \xi_{q i}(y) \xi_{d j}(y)= \\
& \frac{\exp \left(i \theta_{i j}^{d}\right)}{N_{q i} N_{d j}} \int d y \exp \left[F_{q i}\left(\mu_{q i} y+\frac{1}{\tanh \mu_{q i}} \ln \frac{\cosh \mu_{q i}(1-y)}{\cosh \mu_{q i} y \cosh \mu_{q i}}\right)\right. \\
& \left.+F_{d j}\left(\mu_{d j} y+\frac{1}{\tanh \mu_{d j}} \ln \frac{\cosh \mu_{d j}(1-y)}{\cosh \mu_{d j} y \cosh \mu_{d j}}\right)\right],
\end{aligned}
$$

where only $\theta_{12}^{u}, \theta_{23}^{u}, \theta_{12}^{d}, \theta_{23}^{d}$ are the non-zero phases. Indeed, without these phases, the solution satisfying the quark mass ratios and the CKM matrix cannot be obtained [13]. Further, if these phases are small enough, the mass matrices' structure transforms from almost pure-phase $M \approx g_{Y} v / \sqrt{2}\left\{e^{i \theta_{i j}}\right\}$ to almost democratic $M \approx g_{Y} v / \sqrt{2}\{1\}$. All our numerical solutions obtained below indeed clearly reflects this democratic structure. One very important advantage of a DMM is that it has three eigenvalues of "loose hierarchy": $(0$, $0,3 g_{Y} v / \sqrt{2}$ ), and by slightly modifying the mass matrix elements from "1" one can reproduce the right mass spectrum and right CKM matrix. More specifically, because small differences in $F$ 's and $\mu$ 's induce small modification in the corresponding wavefunction profiles $\xi(y)$ 's, 
Eqs. (22), (23), to recover realistic quark masses we will attribute different values of $F_{i}$ and $\mu_{i}$ to different flavors (the quartic coupling $\lambda$ is kept universal). Meanwhile we preserve essential democratic structure by localizing fields from each of the three groups (doublets, up and down-type singlets) at the same point along the extra dimension regardless of family index. Choosing identical signs for $F_{q i}\left(F_{u i}, F_{d i}\right)$ for different indices $i$ one can fulfill this requirement.

Our approach hence is different from that of [9] where wavefunctions of different chiral flavors are very carefully and distinctly constructed in the bulk so that their overlaps render the correct mass spectrum. However, associated CKM matrix found therein does not generate sufficient $\mathrm{CP}$ violation as to the level it is observed in meson rare decays, (as long as the model has only one extra dimensions), even when one assigns to each mass matrix element an arbitrary phase [10]. In the present work a DMM structure will be the key point to overcome this difficulty.

\section{Six-dimensional Model}

With the model with just one extra dimension presented in the previous sections, one can fit quark mass spectrum and CKM matrix all by twisting around the pure-phase and democratic structures of mass matrices. We in particular have employed two different 5d Yukawa couplings $\kappa_{5}^{u}, \kappa_{5}^{d}\left(\kappa_{5}^{u} / \kappa_{5}^{d} \sim 60\right.$, sections IV-V) to generate up-down quark mass hierarchy, whose nature was not seen directly within the framework of 5 -d theory. In this subsection, for the purpose of completeness, we briefly mention a possible solution to this issue, which consists of adding another spatial dimension to the theory.

Beginning with six-dimensional model, we can repeat the orbifold compactification procedure for the two extra dimensions, one after the other, to secure the single-chirality of zero modes. We choose to localize all doublets identically along the sixth dimension (the same holds for up-type and down-type singlets). In the result, the 5-d (now effective) Yukawa coupling $\kappa_{u}^{5}\left(\kappa_{d}^{5}\right)$ are just the product of 6 -d couplings $\kappa_{u}^{6}\left(\kappa_{d}^{6}\right)$ and the $Q-U(Q-D)$ wavefunction overlap along the sixth dimension. So by starting with a single Yukawa coupling $\left(\kappa_{u}^{6}=\kappa_{d}^{6}\right)$ in 6-d theory, we can end up with two different 5-d couplings because $U$ and $D$ fields have been placed differently from $Q$ fields along the sixth dimension. Further, the

phases of mass matrices' elements could also be generated from 6-d models (see details in 
[7, 8]). Rather, the point we would like to emphasize here is that, extra dimension theory can potentially provide necessary ingredients to reproduce 4-d effective theory of particle interaction.

\section{DESCRIPTION OF THE PARAMETER SPACE}

In this section we present the parameter space for the particular choice of the model considered with 24 parameters. Also if the number of parameters is large, what has to be said here is that the "naturality" of the parameters, (generally all the values are of order one or differ by no more than one order of magnitude), we believe, is the most important factor, and the parameter space found satisfies this condition. The list of the parameters is the following:

- $g_{Y u}$ and $g_{Y d}$

- $\mu_{q i}=L \sqrt{\frac{\lambda V_{q i}^{2}}{2}}, \mu_{u i}=L \sqrt{\frac{\lambda V_{u i}^{2}}{2}}$, and $\mu_{d i}=L \sqrt{\frac{\lambda V_{d i}^{2}}{2}}$, with $i=1,2,3$ are dimensionless quantities whose inverse is proportional to the thickness of the domain wall.

- $F_{q i}=\sqrt{2 / \lambda} f_{q i}, F_{u i}=\sqrt{2 / \lambda} f_{u i}$ and $F_{d i}=\sqrt{2 / \lambda} f_{d i}$ with $i=1,2,3$ and $f$ 's being the Yukawa couplings appearing in Eq. (11)

- $\phi_{u 1}, \phi_{u 2}, \phi_{d 1}$, and $\phi_{d 2}$ are the phases appearing respectively in the up and down mass matrices

As it can be seen from this particular choice of the parameter space, we decided to break family symmetry by choosing different values for $\mu_{i}$ and $f_{i}$ for different indices $i$ (toghether with four different phases $\theta_{12}^{u}, \theta_{23}^{u}, \theta_{12}^{d}, \theta_{23}^{d}$ appearing in the mass matrices), and at the same time to break the left-right symmetry by different values for the left component parameters $\mu_{q}$ and $f_{q}$ and the right component parameters $\mu_{u}, f_{u}, \mu_{d}$ and $f_{d}$.

\section{RESULTS FOR MASS MATRICES FROM FIVE DIMENSIONS}

In this section we present the numerical results obtained for the parameter space and for the physical quantities of Table 1 . We consider four different cases, which correspond to the 
all four possible ways of picking the sign of the Yukawa couplings $f$ for the left and right components. The four different cases are the following:

- $f_{q i}>0 f_{u i}>0 f_{d i}>0$ denoted as $(+++)$

- $f_{q i}>0 f_{u i}>0 f_{d i}<0$ denoted as $(++-)$

- $f_{q i}>0 f_{u i}<0 f_{d i}>0$ denoted as $(+-+)$

- $f_{q i}>0 f_{u i}<0 f_{d i}<0$ denoted as $(+--)$

The first case corresponds to the doublets, up and down-type singlets all localized at the orbifold fixed point $y=0$. The second case corresponds to the doublets and up-type singlets localized at $y=0$, while the down-type singlets at $y=L$. The third case corresponds to the doublets and down-type singlets localized at $y=0$, while the up-type singlets at $y=L$. And finally the fourth case corresponds to the doublets localized at $y=0$, while both the up and down-type singlets at $y=L$.

The other four possible cases obtained when one changes at the same time all the signs of the Yukawa couplings are just symmetrical to the four presented, with each wave function now localized at the other orbifold fixed point, and with symmetrical profile. So they do not present any new mixing pattern.

The approach we use to derive the parameter space consists in minimizing a particular function, built in such a way that its global minima correspond to the region defined by the experimental constraints. This function is defined as:

$$
\begin{aligned}
E & =\sum_{i=1}^{N} \frac{\left(x_{i}^{t h}-x_{i}^{\text {min }}\right)^{2}}{<x_{i}>^{2}} \theta\left(x_{i}^{\min }-x_{i}^{t h}\right) \\
& +\sum_{i=1}^{N} \frac{\left(x_{i}^{t h}-x_{i}^{\text {max }}\right)^{2}}{<x_{i}>^{2}} \theta\left(x_{i}^{t h}-x_{i}^{\max }\right)
\end{aligned}
$$

where $\theta(x)$ is the step function, $\mathrm{N}$ is the number of quantities that we want to fit, $x_{i}^{t h}$ is the predicted value for the $i$ th quantity, $x_{i}^{\min }$ and $x_{i}^{\max }$ fix the range for the $i$ th quantity, and $\left\langle x_{i}\right\rangle$ is its average value. It is immediate to verify from Eq. (24) that when all the predicted quantities $x_{i}^{t h}$ 's are contained in the proper ranges, the function $E$ takes its minimum value equal to zero. The set of parameters which correspond to a zero value for the function $E$ is called a solution. 
The minimization procedure we used is called simulated annealing [15] [16], and when the function that we want to minimize depends on many parameters, this procedure seems to work more efficiently than others. In particular the simulated annealing method is mostly used when the global minima are surrounded by a lot of local minima. In fact this minimization process can find a global minimum also after being trapped in a local minimum.

In the following we will present the numerical results for each of the cases mentioned above. We will present graphically together with the parameter space (Figs. 16 1116), the solutions of the mass spectrum (Figs. 381318) (masses are given in GeV and are evaluated at the $M_{Z}$ scale), the CKM matrix (Figs. 4,9,14,19), and the $\bar{\rho}, \bar{\eta}$ CP parameters (Figs. 51015120). For each case we will also give one particular numerical example of all model's parameters, the corresponding mass matrices with eigenvalues (i.e. quark masses), the rotation matrices, the CKM matrix, the $\mathrm{CP}$ parameters, the corresponding plots of background scalar fields and the wavefunction profiles for left and right components (Figs. 271217). Complex phases are measured in radiant.

$$
\text { - } f_{q i}>0 f_{u i}>0 f_{d i}>0
$$

$$
\begin{gathered}
g_{Y u(24)}^{(+++)} \frac{v}{\sqrt{2}}=57.81, \quad g_{Y d(24)}^{(+++)} \frac{v}{\sqrt{2}}=0.98, \\
F_{q 1(24)}^{(+++)}=1.389, \quad F_{q 2(24)}^{(+++)}=0.979, \quad F_{q 3(24)}^{(+++)}=0.787, \\
F_{u 1(24)}^{(+++)}=0.938, \quad F_{u 2(24)}^{(+++)}=0.843, \quad F_{u 3(24)}^{(+++)}=1.352, \\
F_{d 1(24)}^{(+++)}=1.344, \quad F_{d 2(24)}^{(+++)}=1.013, \quad F_{d 3(24)}^{(+++)}=1.437, \\
\mu_{q 1(24)}^{(+++)}=2.252, \mu_{q 2(24)}^{(+++)}=3.367, \mu_{q 3(24)}^{(+++)}=2.660, \\
\mu_{u 1(24)}^{(+++)}=1.965, \quad \mu_{u 2(24)}^{(+++)}=2.060, \mu_{u 3(24)}^{(+++)}=1.496, \\
\mu_{d 1(24)}^{(+++)}=2.537, \mu_{d 2(24)}^{(+++)}=3.157, \quad \mu_{d 3(24)}^{(+++)}=2.520 . \\
\phi_{u 1(24)}^{(+++)}=-0.0001, \phi_{u 2(24)}^{(+++)}=0.0155, \quad \phi_{d 1(24)}^{(+++)}=-0.0095, \quad \phi_{d 2(24)}^{(+++)}=-0.1607, \\
M_{u(24)}^{(+++)}=57.81 G e V\left(\begin{array}{ccc}
0.9814 & 0.9811 e^{-i 0.0001} \\
0.9443 & 0.9438 & 0.9705 \\
0.9938 & 0.9936 & 0.9870
\end{array}\right),
\end{gathered}
$$




$$
\begin{aligned}
& m_{u(24)}^{(+++)}=0.0027 \mathrm{GeV}, \quad m_{c(24)}^{(+++)}=0.677 \mathrm{GeV}, \quad m_{t(24)}^{(+++)}=168.13 \mathrm{GeV}, \\
& M_{d(24)}^{(++)}=0.975 \mathrm{GeV}\left(\begin{array}{ccc}
0.9973 & 0.9934 e^{-i 0.0095} & 0.9955 \\
0.9975 & 0.9996 & 0.9988 e^{-i 0.1607} \\
0.9880 & 0.9808 & 0.9845
\end{array}\right), \\
& m_{d(24)}^{(+++)}=0.0048 \mathrm{GeV}, \quad m_{s(24)}^{(+++)}=0.106 \mathrm{GeV}, \quad m_{b(24)}^{(+++)}=2.90 \mathrm{GeV} .
\end{aligned}
$$

In Eqs. (33), (35) the mass matrices are written in a form that better evidenciates the almost democratic structure. In Eqs. (37) and (38) we give the expressions for the rotation matrices $U_{L}^{u \dagger}$ and $U_{L}^{d}$ whose product is just the CKM matrix, Eq. (39). In Eq. (40) we give the absolute value of the CKM matrix, and in Eq. (41) the values for the CP parameters $\bar{\rho}$ and $\bar{\eta}$ and the invariant area of the unitary triangle $J_{C P}$.

$$
\begin{aligned}
& U_{L(24)}^{u \dagger(+++)}=\left(\begin{array}{ccc}
0.7429 & -0.1027-0.1315 i & -0.6361+0.1249 i \\
-0.3067-0.1223 i & 0.8123 & -0.4664+0.1167 i \\
0.5823 & 0.5588-0.0028 i & 0.5905
\end{array}\right) \text {, } \\
& U_{L(24)}^{d(+++)}=\left(\begin{array}{ccc}
-0.6924+0.0002 i & -0.4298-0.0234 i & 0.5783+0.0292 i \\
-0.0207+0.0031 i & 0.8141 & 0.5803 \\
0.7212 & -0.3893-0.0191 i & 0.5718+0.0306 i
\end{array}\right), \\
& V_{C K M(24)}^{(+++)}=\left(\begin{array}{ccc}
-0.9706+0.0927 i & -0.1529-0.1609 i & 0.0025-0.0027 i \\
-0.1408+0.1713 i & 0.9741+0.0232 i & 0.0274-0.0272 i \\
0.0111+0.0019 i & -0.0252-0.0272 i & 0.9987+0.0334 i
\end{array}\right) \text {, } \\
& \left|V_{C K M(24)}^{(+++)}\right|=\left(\begin{array}{ccc}
0.9750 & 0.2220 & 0.0037 \\
0.2217 & 0.9744 & 0.0386 \\
0.0113 & 0.0371 & 0.9992
\end{array}\right) \\
& \bar{\rho}_{(24)}^{(+++)}=0.28, \quad \bar{\eta}_{(24)}^{(+++)}=0.31, \quad J_{C P(24)}^{(+++)}=-2.2 \times 10^{-5} .
\end{aligned}
$$

with $\bar{\rho}$ and $\bar{\eta}$ defined as

$$
\begin{aligned}
& \bar{\rho}=\operatorname{Re}\left(V_{u d} V_{u b}^{*} V_{c d}^{*} V_{c b}\right) /\left|V_{c d} V_{c b}^{*}\right|^{2}, \\
& \bar{\eta}=\operatorname{Im}\left(V_{u d} V_{u b}^{*} V_{c d}^{*} V_{c b}\right) /\left|V_{c d} V_{c b}^{*}\right|^{2},
\end{aligned}
$$


and $J_{C P}$ as

$$
J_{C P}=\operatorname{Im}\left(V_{u s} V_{u b}^{*} V_{c s}^{*} V_{c b}\right) .
$$

- $f_{q i}>0 f_{u i}>0 f_{d i}<0$

$$
\begin{aligned}
& g_{Y u(24)}^{(++)} \frac{v}{\sqrt{2}}=59.85, \quad g_{Y d(24)}^{(++-)} \frac{v}{\sqrt{2}}=1.18, \\
& F_{q 1(24)}^{(++-)}=0.482, \quad F_{q 2(24)}^{(++-)}=0.888, \quad F_{q 3(24)^{(++-)}}=2.522, \\
& F_{u 1(24)}^{(++-)}=0.966, \quad F_{u 2(24)}^{(++-)}=0.725, \quad F_{u 3(24)}^{(++-)}=1.185, \\
& F_{d 1(24)}^{(++-)}=-1.024, \quad F_{d 2(24)}^{(++-)}=-1.639, \quad F_{d 3(24)}^{(++-)}=-0.014, \\
& \mu_{q 1(24)}^{(++-)}=2.279, \mu_{q 2(24)}^{(++-)}=2.474, \mu_{q 3(24)}^{(++-)}=1.234, \\
& \mu_{u 1(24)}^{(++-)}=1.755, \mu_{u 2(24)}^{(++-)}=2.597, \mu_{u 3(24)}^{(++-)}=2.000, \\
& \mu_{d 1(24)}^{(++-)}=2.223, \mu_{d 2(24)}^{(++-)}=2.079, \mu_{d 3(24)}^{(++-)}=1.482, \\
& \phi_{u 1(24)}^{(++-)}=-0.0007, \quad \phi_{u 2(24)}^{(++-)}=-0.0018, \phi_{d 1(24)}^{(++-)}=0.0122, \phi_{d 2(24)}^{(+++-)}=-0.0951 . \\
& M_{u(24)}^{(++-)}=59.85 \mathrm{GeV}\left(\begin{array}{ccc}
0.9997 & 0.9899 e^{-i 0.0007} & 0.9910 \\
0.9887 & 0.9995 & 0.9992 e^{-i 0.0018} \\
0.9998 & 0.9952 & 0.9960
\end{array}\right), \\
& m_{u(24)}^{(++-)}=0.0024 \mathrm{GeV}, \quad m_{c(24)}^{(++-)}=0.722 \mathrm{GeV}, \quad m_{t(24)}^{(++-)}=178.7 \mathrm{GeV}, \\
& M_{d(24)}^{(++-)}=1.18 \mathrm{GeV}\left(\begin{array}{ccc}
0.8867 & 0.8337 e^{i 0.0122} & 0.9863 \\
0.7955 & 0.7293 & 0.9428 e^{-i 0.0951} \\
0.8656 & 0.8090 & 0.9779
\end{array}\right), \\
& m_{d(24)}^{(++-)}=0.0051 \mathrm{GeV}, \quad m_{s(24)}^{(++-)}=0.082 \mathrm{GeV}, \quad m_{b(24)}^{(++-)}=3.1 \mathrm{GeV} . \\
& U_{L(24)}^{u \dagger(++-)}=\left(\begin{array}{ccc}
-0.5859+0.0191 i & -0.2010-0.0193 i & 0.7846 \\
-0.5691+0.0193 i & 0.7910 & -0.2229-0.0189 i \\
0.5763+0.0001 i & 0.5775+0.0003 i & 0.5783
\end{array}\right) \text {, }
\end{aligned}
$$




$$
\begin{aligned}
& U_{L(24)}^{d(++-)(24)}=\left(\begin{array}{ccc}
-0.6624-0.1144 i & -0.4187-0.1241 i & 0.5979 \\
-0.0511+0.1249 i & 0.8265 & 0.5461-0.0235 i \\
0.7280 & -0.3424+0.0946 i & 0.5863-0.0019 i
\end{array}\right) \\
& V_{C K M(24)}^{(++-)}=\left(\begin{array}{ccc}
0.9741+0.0303 i & -0.1870+0.1230 i & -0.0004+0.0041 i \\
0.1765+0.1373 i & 0.9726+0.0479 i & -0.0390-0.0177 i \\
0.0097+0.0061 i & 0.0380-0.0166 i & 0.9990-0.0144 i
\end{array}\right) \\
& \left|V_{C K M(24)}^{(++-)}\right|=\left(\begin{array}{lll}
0.9746 & 0.2239 & 0.0041 \\
0.2236 & 0.9737 & 0.0428 \\
0.0115 & 0.0415 & 0.9991
\end{array}\right) \\
& \bar{\rho}_{(24)}^{(++-)}=0.13, \quad \bar{\eta}_{(24)}^{(++-)}=0.40, \quad J_{C P(24)}^{(++-)}=-3.6 \times 10^{-5} .
\end{aligned}
$$

- $f_{q i}>0 f_{u i}<0 f_{d i}>0$

$$
\begin{aligned}
& g_{Y u(24)}^{(+-+)} \frac{v}{\sqrt{2}}=61.66, \quad g_{Y d(24)}^{(+-+)} \frac{v}{\sqrt{2}}=1.00, \\
& F_{q 1(24)}^{(+-+)}=3.152, \quad F_{q 2(24)}^{(+-+)}=0.327, F_{q 3(24)}^{(+-+)}=0.485, \\
& F_{u 1(24)}^{(+-+)}=-0.112, \quad F_{u 2(24)}^{(+-+)}=-0.629, \quad F_{u 3(24)}^{(+-+)}=-0.516, \\
& F_{d 1(24)}^{(+-+)}=1.062, \quad F_{d 2(24)}^{(+-+)}=0.087, \quad F_{d 3(24)}^{(+-+)}=1.244, \\
& \mu_{q 1(24)}^{(+-+)}=1.467, \mu_{q 2(24)}^{(+-+)}=2.142, \quad \mu_{q 3(24)}^{(+-+)}=2.458, \\
& \mu_{u 1(24)}^{(+-+)}=1.942, \quad \mu_{u 2(24)}^{(+-+)}=1.658, \quad \mu_{u 3(24)}^{(+-+)}=1.452, \\
& \mu_{d 1(24)}^{(+-+)}=2.008, \mu_{d 2(24)}^{(+-+)}=1.820, \mu_{d 3(24)}^{(+-+)}=2.460, \\
& \phi_{u 1(24)}^{(+-+)}=-0.0006, \phi_{u 2(24)}^{(+-+)}=-0.0000, \phi_{d 1(24)}^{(+-+)}=0.0870, \phi_{d 2(24)}^{(+-+)}=-0.0035 \text {, } \\
& M_{u(24)}^{(+-+)}=61.66 \mathrm{GeV}\left(\begin{array}{ccc}
0.9185 & 0.8840 e^{-i 0.0006} & 0.9032 \\
0.9920 & 0.9785 & 0.9865 e^{-i 0.0000} \\
0.9763 & 0.9557 & 0.9676
\end{array}\right) \\
& m_{u(24)}^{(+-+)}=0.0024 \mathrm{GeV}, \quad m_{c(24)}^{(+-+)}=0.724 \mathrm{GeV}, \quad m_{t(24)}^{(+-+)}=176.1 \mathrm{GeV},
\end{aligned}
$$




$$
\begin{aligned}
& M_{d(24)}^{(+-+)}=1.00 \mathrm{GeV}\left(\begin{array}{ccc}
0.9941 & 0.9357 e^{i 0.0870} & 0.9977 \\
0.9851 & 0.9968 & 0.9403 e^{-i 0.0035} \\
0.9967 & 0.9853 & 0.9672
\end{array}\right) \\
& m_{d(24)}^{(+-+)}=0.0048 \mathrm{GeV}, \quad m_{s(24)}^{(+-+)}=0.082 \mathrm{GeV}, \quad m_{b(24)}^{(+-+)}=2.9 \mathrm{GeV} . \\
& U_{L(24)}^{u \dagger(+-+)}=\left(\begin{array}{ccc}
-0.2669+0.0067 i & -0.5391-0.0062 i & 0.7988 \\
0.7934 & -0.5933-0.0066 i & -0.1354+0.0069 i \\
0.5470+0.0001 i & 0.5977 & 0.5861
\end{array}\right) \\
& U_{L(24)}^{d(+-+)}=\left(\begin{array}{ccc}
-0.1495+0.1160 i & 0.7951 & 0.5759 \\
-0.6160-0.1196 i & -0.5151-0.1001 i & 0.5753-0.0008 i \\
0.7553 & -0.2785+0.1220 i & 0.5806
\end{array}\right) \\
& V_{C K M(24)}^{(+-+)}=\left(\begin{array}{ccc}
0.9738+0.0363 i & -0.1576+0.1600 i & -0.0002-0.0036 i \\
0.1437+0.1723 i & 0.9727+0.0444 i & 0.0370+0.0135 i \\
-0.0073-0.0081 i & -0.0362+0.0118 i & 0.9992+0.0084 i
\end{array}\right) \\
& \left|V_{C K M(24)}^{(+-+)}\right|=\left(\begin{array}{lll}
0.9744 & 0.2246 & 0.0036 \\
0.2244 & 0.9737 & 0.0394 \\
0.0108 & 0.0381 & 0.9992
\end{array}\right) \\
& \bar{\rho}_{(24)}^{(+-+)}=0.17, \quad \bar{\eta}_{(24)}^{(+-+)}=0.36, \quad J_{C P(24)}^{(+-+)}=-2.8 \times 10^{-5} .
\end{aligned}
$$

- $f_{q i}>0 f_{u i}<0 f_{d i}<0$

$$
\begin{gathered}
g_{Y u(24)}^{(+--)} \frac{v}{\sqrt{2}}=60.83, \quad g_{Y d(24)}^{(+--)} \frac{v}{\sqrt{2}}=1.25, \\
F_{q 1(24)}^{(+--)}=2.233, \quad F_{q 2(24)}^{(+--)}=0.357, \quad F_{q 3(24)}^{(+--)}=1.069, \\
F_{u 1(24)}^{(+--)}=-0.429, \quad F_{u 2(24)}^{(+--)}=-1.476, \quad F_{u 3(24)}^{(+--)}=-0.313, \\
F_{d 1(24)}^{(+--)}=-0.323, \quad F_{d 2(24)}^{(+--)}=-0.847, \quad F_{d 3(24)}^{(+--)}=-2.480, \\
\mu_{q 1(24)}^{(+--)}=2.087, \quad \mu_{q 2(24)}^{(+--)}=2.236, \quad \mu_{q 3(24)}^{(+--)}=2.130,
\end{gathered}
$$




$$
\begin{aligned}
& \mu_{u 1(24)}^{(+--)}=1.126, \quad \mu_{u 2(24)}^{(+--)}=1.006, \quad \mu_{u 3(24)}^{(+--)}=1.322 \\
& \mu_{d 1(24)}^{(+--)}=3.380, \quad \mu_{d 2(24)}^{(+--)}=1.736, \quad \mu_{d 3(24)}^{(+--)}=1.693 .
\end{aligned}
$$$$
\phi_{u 1(24)}^{(+--)}=-0.0053, \phi_{u 2(24)}^{(+--)}=-0.0003, \quad \phi_{d 1(24)}^{(+--)}=0.0218, \quad \phi_{d 2(24)}^{(+--)}=-0.0525,
$$$$
M_{u(24)}^{(+-)}=60.83 \mathrm{GeV}\left(\begin{array}{ccc}
0.8427 & 0.8188 e^{-i 0.0053} & 0.8412 \\
0.9893 & 0.9819 & 0.9889 e^{-i 0.0003} \\
0.9447 & 0.9293 & 0.9437
\end{array}\right),
$$$$
m_{u(24)}^{(+--)}=0.0022 \mathrm{GeV}, \quad m_{c(24)}^{(+--)}=0.677 \mathrm{GeV}, \quad m_{t(24)}^{(+--)}=168.3 \mathrm{GeV},
$$$$
M_{d(24)}^{(+--)}=1.25 G e V\left(\begin{array}{ccc}
0.7298 & 0.7668 e^{i 0.0218} & 0.5944 \\
0.9438 & 0.9613 & 0.8640 e^{-i 0.0525} \\
0.8664 & 0.8936 & 0.7580
\end{array}\right)
$$$$
m_{d(24)}^{(+--)}=0.0050 \mathrm{GeV}, \quad m_{s(24)}^{(+--)}=0.081 \mathrm{GeV}, \quad m_{b(24)}^{(+--)}=3.1 \mathrm{GeV} .
$$

$$
U_{L(24)}^{u \dagger(+-)}=\left(\begin{array}{ccc}
-0.3525+0.0856 i & -0.4668-0.0730 i & 0.8033 i \\
0.7718 & -0.6230-0.0844 i & -0.0311+0.0898 i \\
0.5223+0.0008 i & 0.6177 & 0.5880-0.0001 i
\end{array}\right)
$$

$$
\begin{aligned}
& U_{L(24)}^{d(+-)}=\left(\begin{array}{ccc}
-0.4256-0.2346 i & 0.7244 & 0.4889+0.0110 i \\
-0.3737+0.1807 i & -0.5963+0.2371 i & 0.6449 \\
0.7691 & 0.0554-0.2459 i & 0.5873+0.0085 i
\end{array}\right) \\
& V_{C K M(24)}^{(+--)}=\left(\begin{array}{ccc}
0.9755-0.0108 i & 0.0848-0.2026 i & -0.0025-0.0022 i \\
-0.1043-0.1930 i & 0.9710-0.0847 i & -0.0435+0.0065 i \\
-0.0007-0.0113 i & 0.0426+0.0024 i & 0.9990+0.0112 i
\end{array}\right) \text {, } \\
& \left|V_{C K M(24)}^{(+--)}\right|=\left(\begin{array}{lll}
0.9756 & 0.2197 & 0.0034 \\
0.2194 & 0.9746 & 0.0440 \\
0.0113 & 0.0426 & 0.9990
\end{array}\right) \\
& \bar{\rho}_{(24)}^{(+--)}=0.13, \quad \bar{\eta}_{(24)}^{(+--)}=0.31, \quad J_{C P(24)}^{(+--)}=-2.9 \times 10^{-5} .
\end{aligned}
$$

By looking at the four cases one can notice that all mass matrices have almost democratic structure with deviations from democracy for the up and down sector which depend on the 
different cases. In particular the situation with all components localized at the same orbifold fixed point $(+++)$ has both mass matrices very close to a DMM. The mass matrices, except the different Yukawa prefactors, are very similar. In this case a small top mass seems to be favored (Fig. 3). For the configuration with the doublets localized at the zero orbifold fixed point and both the up and down-singlets at the other orbifold fixed point $(+--)$, the deviations from a pure democratic mass matrix are large for both mass matrices. Also in this case a small top mass seems to be favored (Fig. 18). The situation is different in the other two cases where the up and down-type singlets are localized at different orbifold fixed points. In particular the case with the doublets and down-type singlets right components localized at the zero fixed point and the up-type singlets at the other orbifold fixed point $(+-+)$ seems to be the one which allows a larger range for the top-quark mass values (Fig. 13). In this case the deviation from a pure democratic mass matrix for the up sector is bigger than the one for the down sector. As we will show in the next section this is also the only case for which we were able to find solutions for the 15 parameter version of the model. The forth case where the doublets and up-type singlets are at the same orbifold fixed point while the down-type singlets are at the other orbifold fixed point $(++-)$ gives for the top-quark mass a very narrow value-region around $178 \mathrm{GeV}$ (Fig. 8). In this case the mass matrix for the up sector is very close to a pure democratic mass matrix while the deviation from it is larger for the down sector. What is important to say here is that by looking at the four different cases, it seems that deviation from a DMM are bigger when left and right components are localized at different orbifold fixed points.

\section{15 PARAMETER VERSION}

In this section we present the results for another particular choice of the model with 15 parameters, which correspond to all the Yukawa couplings with the same absolute value, $\left|F_{q, i}\right|=\left|F_{u, i}\right|=\left|F_{d, i}\right|=1$. The family symmetry and left-right symmetry are now broken only through the parameters $\mu$ 's and phases $\theta$ 's. The important point is that for the 15 parameter model choice we were able to find solutions only for the case corresponding to $f_{q}>0, f_{u}<0, f_{d}>0$ with the conditions that all $\mu$ 's are bigger than one (6). In the other three cases we were not be able to find solutions if we decided to keep the constraints $\mu^{\prime} s>1$. The fact that we found solutions only for one of the four possible cases does not 
obviously exclude completely the existence of solutions for the other three cases, but we believe that we can at least conclude that the configuration $f_{q}>0, f_{u}<0, f_{d}>0$ is favored respect to the others.

In the following we give the solutions for the model's parameters and physics quantities as in the cases of 24 parameter version (see also Figs. 21]25)

- $F_{q, i}=1 F_{u, i}=-1 F_{d, i}=1$

$$
\begin{aligned}
& g_{Y u(15)}^{(+-+)} \frac{v}{\sqrt{2}}=60.69, \quad g_{Y d(15)}^{(+-+)} \frac{v}{\sqrt{2}}=1.08 \\
& \mu_{q 1(15)}^{(+-+)}=2.513, \mu_{q 2(15)}^{(+-+)}=1.928, \mu_{q 3(15)}^{(+-+)}=1.993, \\
& \mu_{u 1(15)}^{(+-+)}=1.177, \mu_{u 2(15)}^{(+-+)}=1.562, \mu_{u 3(15)}^{(+-+)}=1.152, \\
& \mu_{d 1(15)}^{(+-+)}=4.969, \mu_{d 2(15)}^{(+-+)}=5.427, \mu_{d 3(15)}^{(+-+)}=1.022 . \\
& \phi_{u 1(15)}^{(+-+)}=0.0153, \quad \phi_{u 2(15)}^{(+-+)}=-0.0001, \quad \phi_{d 1(15)}^{(+-+)}=-0.0423, \quad \phi_{d 2(15)}^{(+-+)}=-0.0279, \\
& M_{u(15)}^{(+-+)}=60.69 G e V\left(\begin{array}{ccc}
0.8968 & 0.8619 e^{i 0.0153} & 0.8986 \\
0.9520 & 0.9267 & 0.9532 e^{-i 0.0001} \\
0.9469 & 0.9204 & 0.9481
\end{array}\right) \\
& m_{u(15)}^{(+-+)}=0.0024 \mathrm{GeV}, \quad m_{c(15)}^{(+-+)}=0.713 \mathrm{GeV}, \quad m_{t(15)}^{(+-+)}=168.1 \mathrm{GeV} \\
& M_{d(15)}^{(+-+)}=1.08 \mathrm{GeV}\left(\begin{array}{ccc}
0.9086 & 0.8874 e^{-i 0.0423} & 0.9450 \\
0.8414 & 0.8158 & 0.9829 e^{-i 0.0279} \\
0.8496 & 0.8245 & 0.9798
\end{array}\right) \\
& m_{d(15)}^{(+-+)}=0.0052 \mathrm{GeV}, \quad m_{s(15)}^{(+-+)}=0.084 \mathrm{GeV}, \quad m_{b(15)}^{(+-+)}=2.9 \mathrm{GeV} . \\
& U_{L(15)}^{u \dagger(+-+)}=\left(\begin{array}{ccc}
-0.0336-0.0417 i & -0.6876+0.0393 i & 0.7231 \\
0.8308 & -0.4195+0.0342 i & -0.3622-0.0382 i \\
0.5540-0.0027 i & 0.5904 & 0.5869
\end{array}\right) \\
& U_{L(15)}^{d(+-+)}=\left(\begin{array}{ccc}
-0.1221-0.1091 i & 0.7910 & 0.5895 \\
-0.6283+0.1216 i & -0.5048+0.1047 i & 0.5698+0.0010 i \\
0.7508 & -0.3108-0.1091 i & 0.5725+0.0074 i
\end{array}\right)
\end{aligned}
$$




$$
\begin{gathered}
V_{C K M(15)}^{(+-+)}=\left(\begin{array}{ccc}
0.9696-0.0995 i & 0.0917-0.2038 i & 0.0024+0.0025 i \\
-0.1140-0.1919 i & 0.9737-0.0098 i & 0.0436-0.0055 i \\
0.0018+0.0116 i & -0.0422-0.0044 i & 0.9990+0.0033 i
\end{array}\right) \\
\left|V_{C K M(15)}^{(+-+)}\right|=\left(\begin{array}{ccc}
0.9747 & 0.2235 & 0.0034 \\
0.2232 & 0.9738 & 0.0439 \\
0.0118 & 0.0424 & 0.9990
\end{array}\right) \\
\bar{\rho}_{(15)}^{(+-+)}=0.16, \quad \bar{\eta}_{(15)}^{(+-+)}=0.30, \quad J_{C P(15)}^{(+-+)}=-2.9 \times 10^{-5}
\end{gathered}
$$

As it can be seen in the numerical example given above, also in the case of the 15 parameter version, as for all the 24 parameter cases, both mass matrices for the up and down sector are almost democratic. What has to be also noticed is that the 15 parameter case favors a small top mass (Fig. 23), on the contrary to the 24 parameter corresponding case $(+-+)$ which gives a much larger range for the top-quark mass.

\section{EPILOGUE}

We suggest that using one extra dimension compactified on an $S_{1} / Z_{2}$ orbifold one is able to produce an almost democratic mass matrix and obtain the right mass spectrum and right CKM matrix. In the model presented the zero modes are localized only at the orbifold fixed points and different profiles for the zero mode wave functions are allowed. We show that in the case of the 24 parameter version of the model, for all four possible scenarios to localize the left and right handed components of quarks at one or the other orbifold fixed point, we were able to fit the mass spectrum and CKM matrix. On the other hand in the case of the 15 parameter version of the model, which corresponds to having the universal absolute value of the Yukawa couplings with the background scalar field for the different fermion families, we were able to reproduce the right mass spectrum and right CKM only in the case with the doublets and down-type singlets localized at one orbifold fixed point and the up-type singlets at the other orbifold fixed point. Finally we just also explain how the existence of a sixth dimension could account for the different Yukawa couplings for the up and down sectors. 


\section{Acknowledgments}

We would like to thank Prof. P.Q. Hung and Dr. M. Seco for valuable discussions, and the University of Virginia High Energy Theory Group for supporting our work. 
[1] H. Georgi, A.K. Grant and G. Hailu, Phys. Rev. D 63, 064027 (2001), (hep-ph/0007350).

[2] R. Jakiw and S. Rebbi, Phys. Rev. D 13, 3398 (1976)

[3] N. Arkani-Hamed and M. Schmaltz, Phys. Rev. D 61, 033005 (2000), (hep-ph/9903417).

[4] A. Masiero, M. Peloso, L. Sorbo and R. Tabbash, Phys. Rev. D 62, 063515 (2000), (hep-ph/0003312).

[5] D. E. Kaplan and T. M. P. Tait, JHEP 11, 051 (2001), (hep-ph/0110126).

[6] Y. Grossman, and G. Perez, Phys. Rev. D 67, 015011 (2001), (hep-ph/0210053).

[7] P. Q. Hung, M. Seco, Nucl. Phys. B 653, 123 (2003), (hep-ph/0111013).

[8] P. Q. Hung, M. Seco and A. Soddu, in preparation; A. Soddu, PhD Thesis, University of Virginia (2003).

[9] E. A. Mirabelli and M. Schmaltz, Phys. Rev. D 61, 113011 (2000), (hep-ph/9912265).

[10] G. C. Branco, A. de Gouvea and M. N. Rebelo, Phys. Lett. B 506, 115 (2001), (hep-ph/0012289).

[11] F. del Aguila and J. Santiago, JHEP 03, 010 (2002), (hep-ph/0111047).

[12] W.-F. Chang and J. N. Ng, JHEP 12, 077 (2002), (hep-ph/0210414).

[13] T. Teshima and T. Sakai, Prog. Theor. Phys. 97, 653 (1997), (hep-ph/9608447).

[14] A. Muck, A. Pilaftsis and R. Ruckl, Phys.Rev. D 65, 085037 (2002), (hep-ph/0110391).

[15] S. Kirkpatrick, C. D. Gelatt, M. P. Vecchi, Science 220, 671-680 (1983).

[16] S. Kirkpatrick, Journal of Statistical Physics 34, 975-986 (1984).

[17] N. Metropolis, A. Rosenbluth. M. Rosenbluth, A. Teller, E. Teller, Journal of Chemical Physics 21, 1087-1092 (1953).

[18] NUMERICAL RECIPES in C, SECOND EDITION, W. H. Press, S. A. Teukolosky, W. T. Vetterling, B. P. Flannery, CAMBRIDGE.

[19] A bare mass term of fermion is forbidden by this symmetry.

[20] As long as $v / \sqrt{2} \simeq 175 \mathrm{GeV}<1 / L$, the Higgs zero mode is the only mode that receives non-zero VEV [14]

[21] By rotating the right-handed quark fields one can absorb 3 phases from each matrix, bringing to 12 the total number of phases. 
TABLE I: Central values and uncertainties for the masses of the 6 quarks evaluated at $M_{Z}$, for the two ratios $m_{u} / m_{d}$ and $m_{s} / m_{d}$, for the absolute values of the CKM matrix elements and the CP parameters $\bar{\rho}, \bar{\eta}$

\begin{tabular}{|c|c|c|}
\hline$x_{i}$ & $<x_{i}>$ & $\left|x_{i}^{\max }-x_{i}^{\min }\right| / 2$ \\
\hline$m_{u}$ & $2.33 \times 10^{-3}$ & $0.45 \times 10^{-3}$ \\
\hline$m_{c}$ & 0.685 & 0.061 \\
\hline$m_{t}$ & 181 & 13 \\
\hline$m_{d}$ & $4.69 \times 10^{-3}$ & $0.66 \times 10^{-3}$ \\
\hline$m_{s}$ & 0.0934 & 0.0130 \\
\hline$m_{b}$ & 3.00 & 0.11 \\
\hline$m_{u} / m_{d}$ & 0.497 & 0.119 \\
\hline$m_{s} / m_{d}$ & 19.9 & 3.9 \\
\hline$\left|V_{u d}\right|$ & 0.97485 & 0.00075 \\
\hline$\left|V_{u s}\right|$ & 0.2225 & 0.0035 \\
\hline$\left|V_{u b}\right|$ & 0.00365 & 0.0115 \\
\hline$\left|V_{c d}\right|$ & 0.2225 & 0.0035 \\
\hline$\left|V_{c s}\right|$ & 0.9740 & 0.0008 \\
\hline$\left|V_{c b}\right|$ & 0.041 & 0.003 \\
\hline$\left|V_{t d}\right|$ & 0.009 & 0.005 \\
\hline$\left|V_{t s}\right|$ & 0.0405 & 0.0035 \\
\hline$\left|V_{t b}\right|$ & 0.99915 & 0.00015 \\
\hline $\bar{\rho}$ & 0.22 & 0.10 \\
\hline $\bar{\eta}$ & 0.35 & 0.05 \\
\hline
\end{tabular}


FIG. 1: Summary of the 24 parameter space corresponding to $f_{q i}>0 f_{u i}>0 f_{d i}>0$.

FIG. 2: Profile of the VEV's and of the wave functions for left and right components corresponding to $f_{q i}>0 f_{u i}>0 f_{d i}>0$ for the 24 parameter space.

FIG. 3: Solutions for the 6 quark masses corresponding to $f_{q i}>0 f_{u i}>0 f_{d i}>0$ for the 24 parameter space. The masses in $G e V$ are evaluated at the $M_{Z}$ scale. The range for each mass is given by the edges of the corresponding window.

FIG. 4: Solutions for the absolute values of the CKM matrix elements corresponding to $f_{q i}>$ $0 f_{u i}>0 f_{d i}>0$ for the 24 parameter space. The range for each element is given by the edges of the window.

FIG. 5: Solutions for $\bar{\rho}$ and $\bar{\eta}$ corresponding to $f_{q i}>0 f_{u i}>0 f_{d i}>0$ for the 24 parameter space. The delimited area is the allowed region in the $\bar{\rho}, \bar{\eta}$ plane.

FIG. 6: Summary of the 24 parameter space corresponding to $f_{q i}>0 f_{u i}>0 f_{d i}<0$.

FIG. 7: Profile of the VEV's and of the wave functions for left and right components corresponding to $f_{q i}>0 f_{u i}>0 f_{d i}<0$ for the 24 parameter case.

FIG. 8: Solutions for the 6 quark masses corresponding to $f_{q i}>0 f_{u i}>0 f_{d i}<0$ for the 24 parameter case. The masses in $G e V$ are evaluated at the $M_{Z}$ scale. The range for each mass is given by the edges of the corresponding window. 
FIG. 9: Solutions for the absolute values of the CKM matrix elements corresponding to $f_{q i}>$ $0 f_{u i}>0 f_{d i}<0$ for the 24 parameter case. The range for each element is given by the edges of the window.

FIG. 10: Solutions for $\bar{\rho}$ and $\bar{\eta}$ corresponding to $f_{q i}>0 f_{u i}>0 f_{d i}<0$ for the 24 parameter case. The delimited area is the allowed region in the $\bar{\rho}, \bar{\eta}$ plane.

FIG. 11: Summary of the 24 parameter space corresponding to $f_{q i}>0 f_{u i}<0 f_{d i}>0$.

FIG. 12: Profile of the VEV's and of the wave functions for left and right components corresponding to $f_{q i}>0 f_{u i}<0 f_{d i}>0$.

FIG. 13: Solutions for the 6 quark masses corresponding to $f_{q i}>0 f_{u i}<0 f_{d i}>0$ for the 24 parameter case. The masses in $G e V$ are evaluated at the $M_{Z}$ scale. The range for each mass is given by the edges of the corresponding window.

FIG. 14: Solutions for the absolute values of the CKM matrix elements corresponding to $f_{q i}>$ $0 f_{u i}<0 f_{d i}>0$ for the 24 parameter case. The range for each element is given by the edges of the window.

FIG. 15: Solutions for $\bar{\rho}$ and $\bar{\eta}$ corresponding to $f_{q i}>0 f_{u i}<0 f_{d i}>0$ for the 24 parameter case. The delimited area is the allowed region in the $\bar{\rho}, \bar{\eta}$ plane.

FIG. 16: Summary of the 24 parameter space corresponding to $f_{q i}>0 f_{u i}<0 f_{d i}<0$.

FIG. 17: Profile of the VEV's and of the wave functions for left and right components corresponding to $f_{q i}>0 f_{u i}<0 f_{d i}<0$ for the 24 parameter case.

FIG. 18: Solutions for the 6 quark masses corresponding to $f_{q i}>0 f_{u i}<0 f_{d i}<0$ for the 24 parameter case. The masses in $G e V$ are evaluated at the $M_{Z}$ scale. The range for each mass is given by the edges of the corresponding window. 
FIG. 19: Solutions for the absolute values of the CKM matrix elements corresponding to $f_{q i}>$ $0 f_{u i}<0 f_{d i}<0$ for the 24 parameter case. The range for each element is given by the edges of the window.

FIG. 20: Solutions for $\bar{\rho}$ and $\bar{\eta}$ corresponding to $f_{q i}>0 f_{u i}<0 f_{d i}<0$ for the 24 parameter case. The delimited area is the allowed region in the $\bar{\rho}, \bar{\eta}$ plane.

FIG. 21: Summary of the 15 parameter space corresponding to $f_{q i}>0 f_{u i}<0 f_{d i}<0$.

FIG. 22: Profile of the VEV's and of the wave functions for left and right components corresponding to $f_{q i}>0 f_{u i}<0 f_{d i}<0$ for the 15 parameter case.

FIG. 23: Solutions for the 6 quark masses corresponding to $f_{q i}>0 f_{u i}<0 f_{d i}<0$ for the 15 parameter case. The masses in $G e V$ are evaluated at the $M_{Z}$ scale. The range for each mass is given by the edges of the corresponding window.

FIG. 24: Solutions for the absolute values of the CKM matrix elements corresponding to $f_{q i}>$ $0 f_{u i}<0 f_{d i}<0$ for the 15 parameter case. The range for each element is given by the edges of the window.

FIG. 25: Solutions for $\bar{\rho}$ and $\bar{\eta}$ corresponding to $f_{q i}>0 f_{u i}<0 f_{d i}<0$ for the 15 parameter case. The delimited area is the allowed region in the $\bar{\rho}, \bar{\eta}$ plane. 

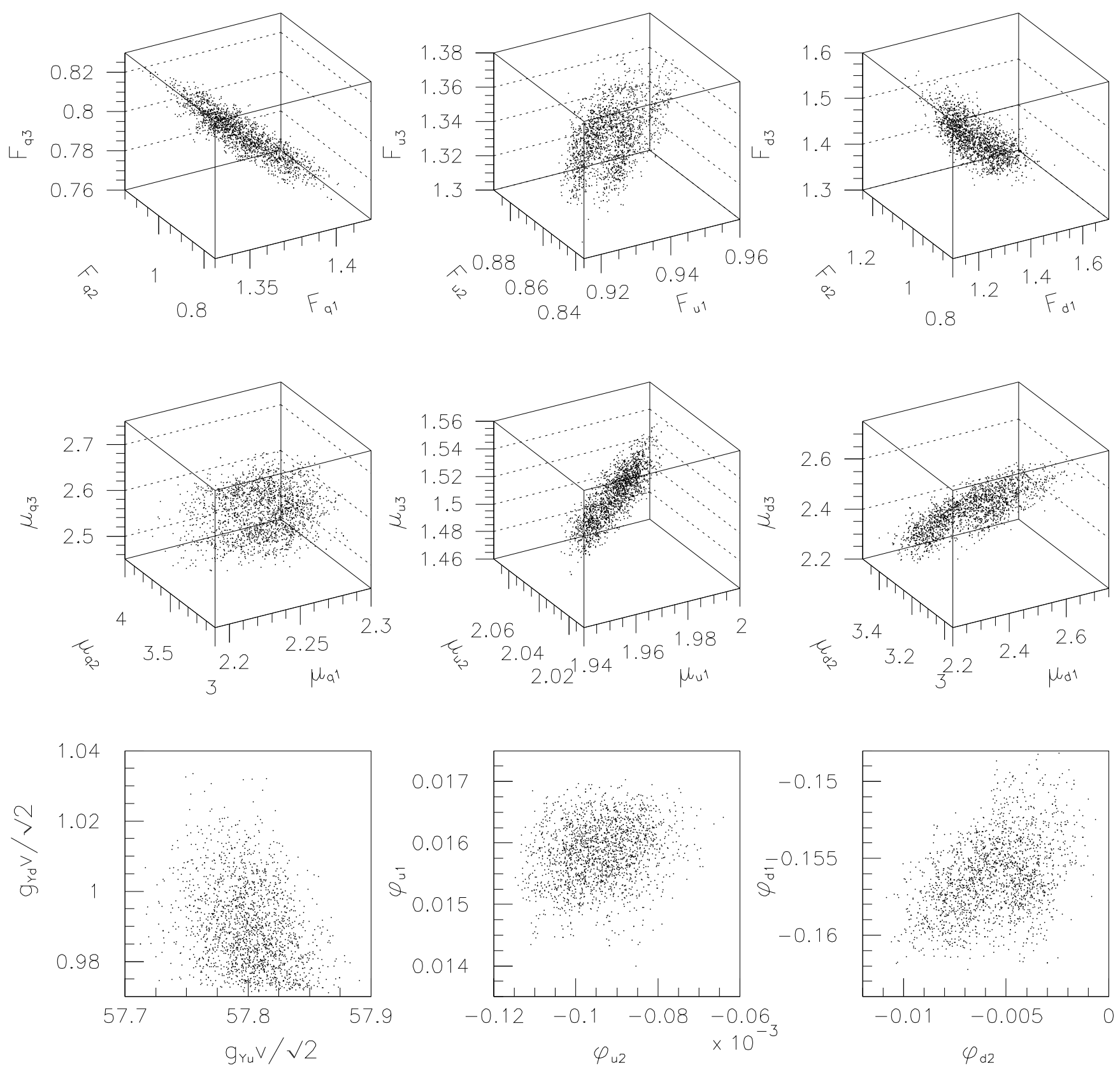

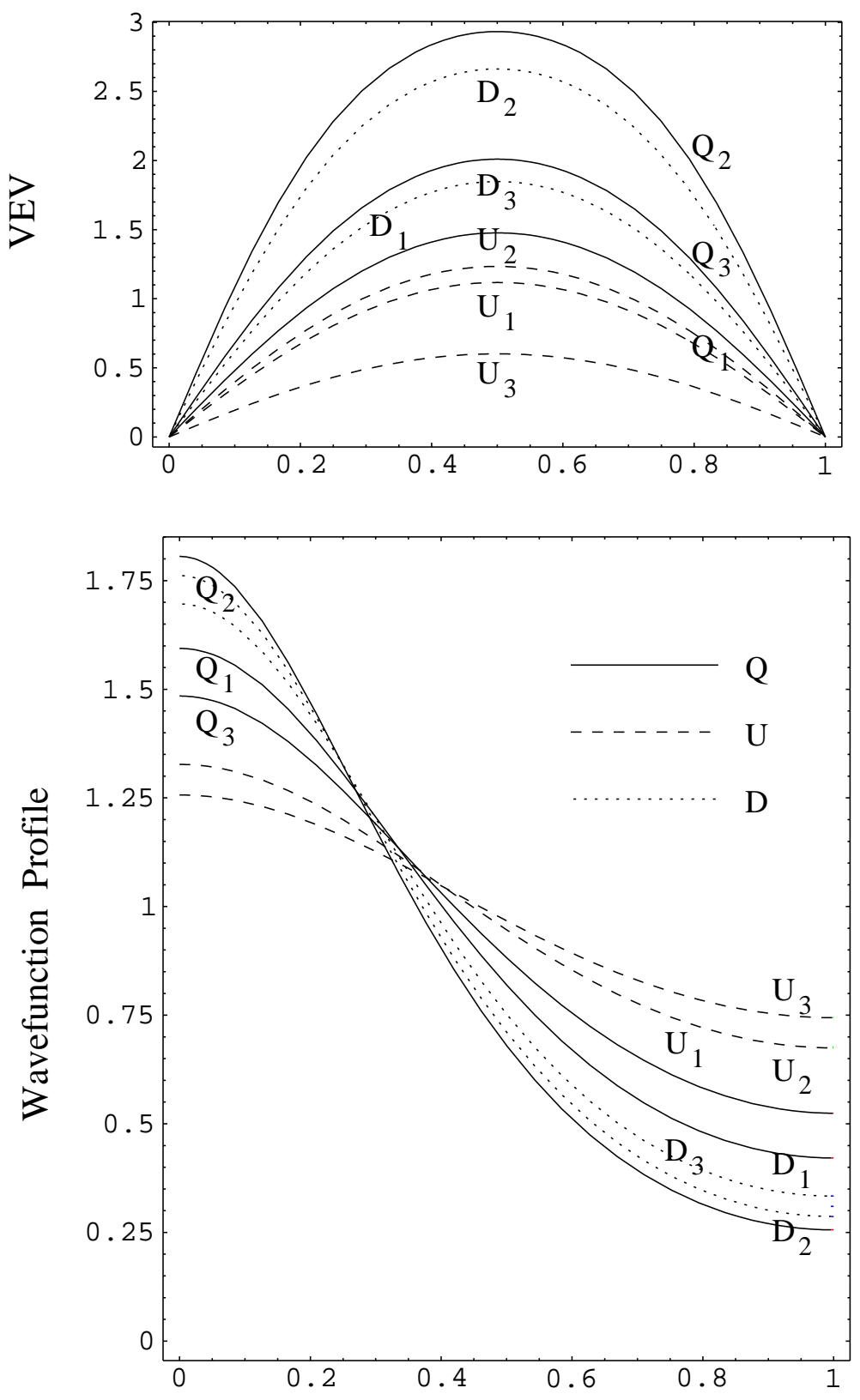

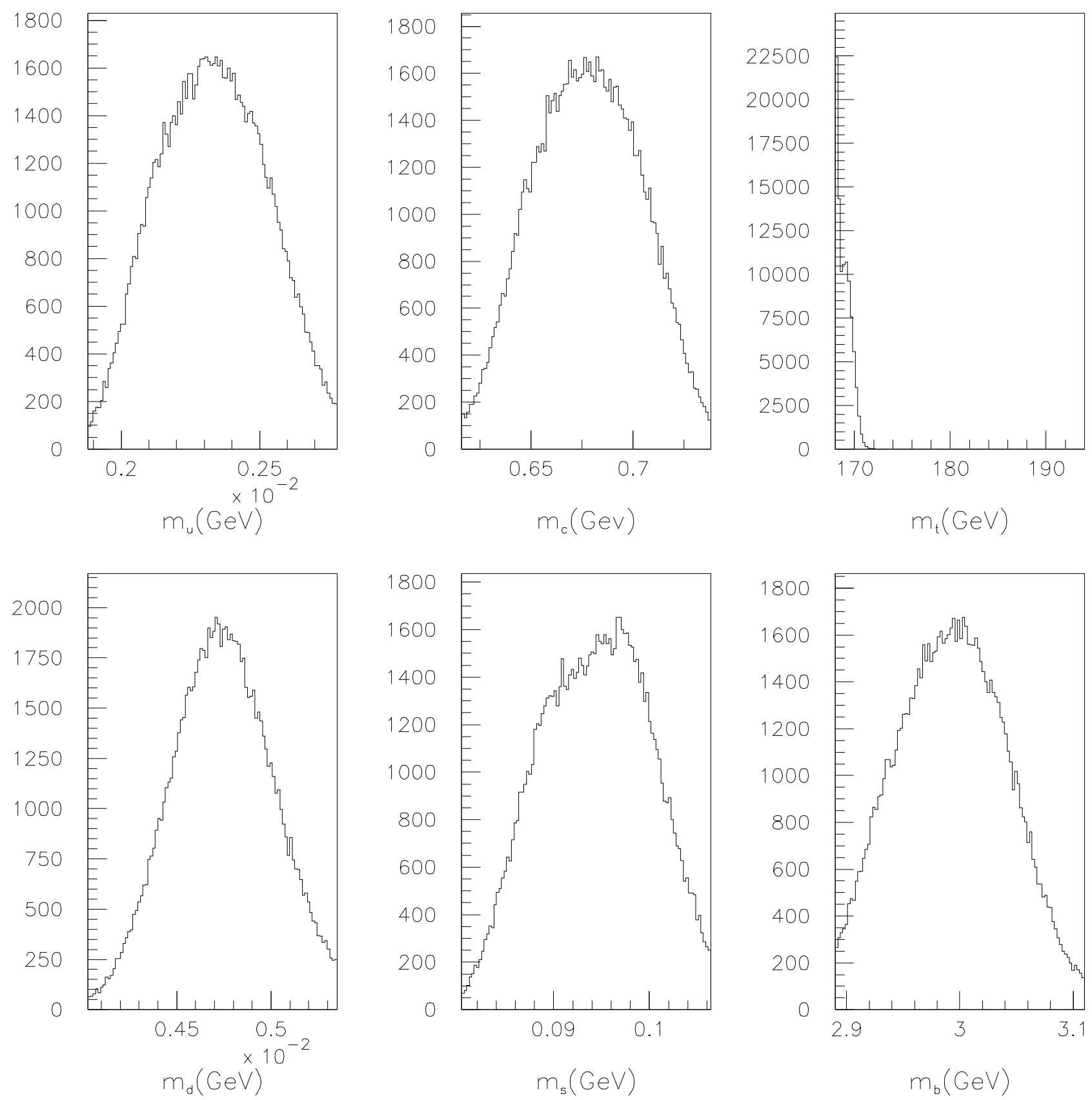

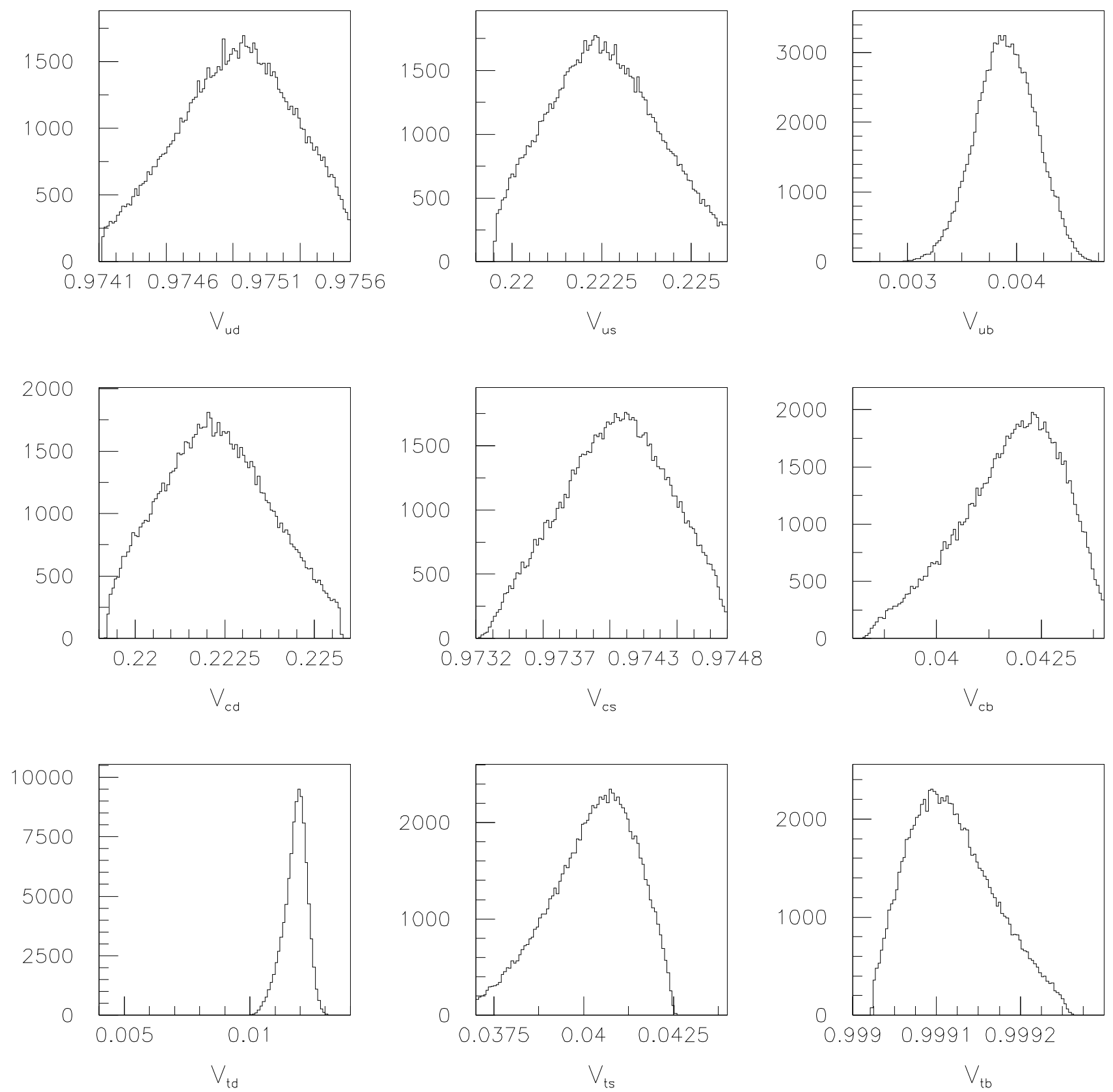


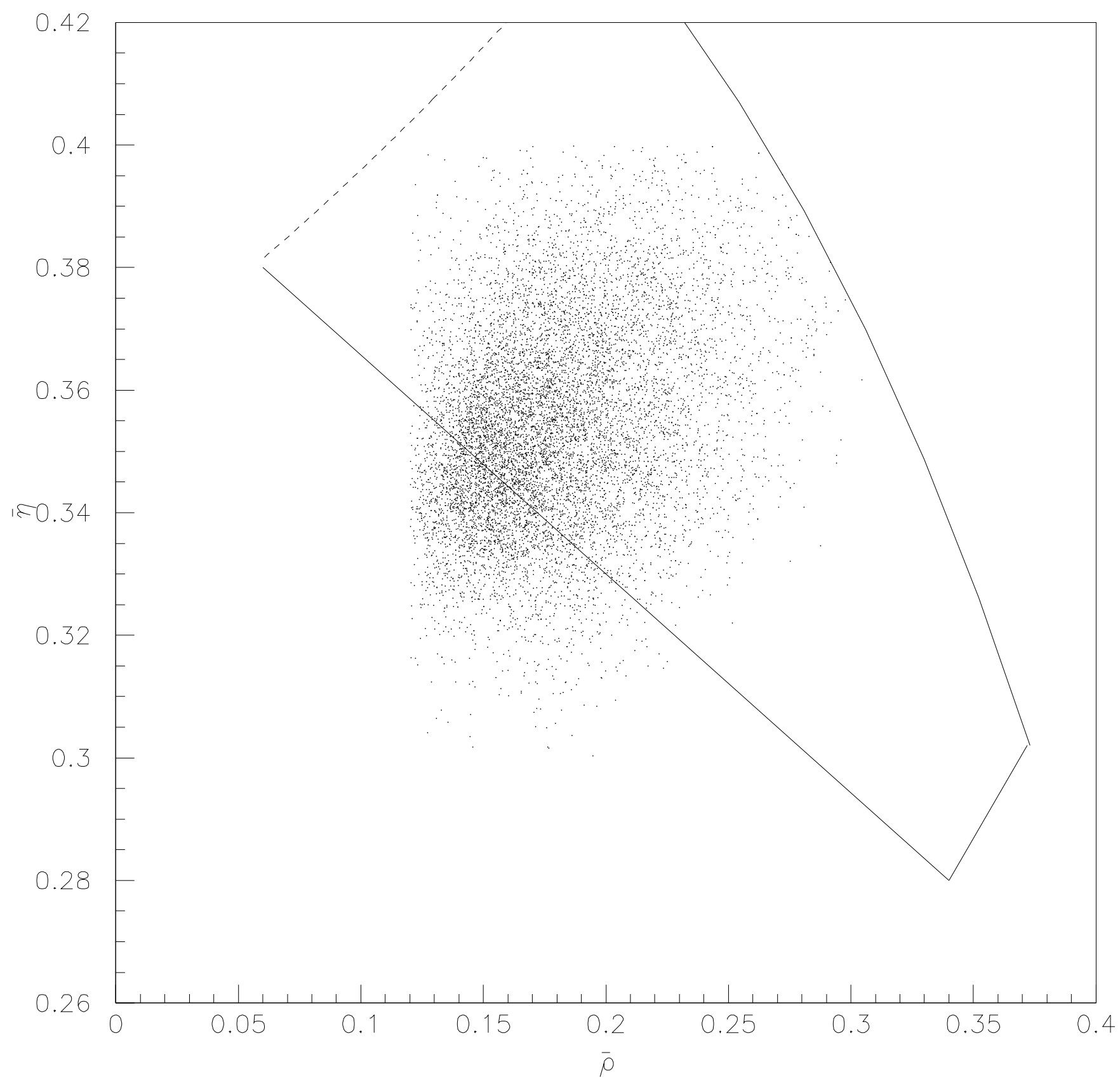



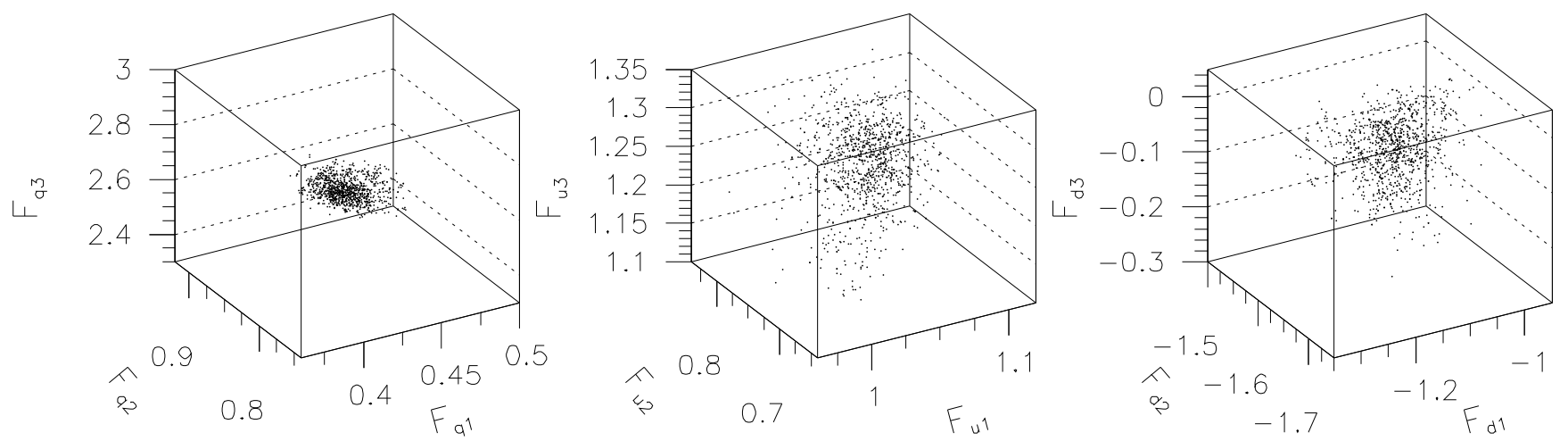

$\begin{array}{lll}o_{2} & -1.7 \quad F_{\mathrm{d} 1}\end{array}$
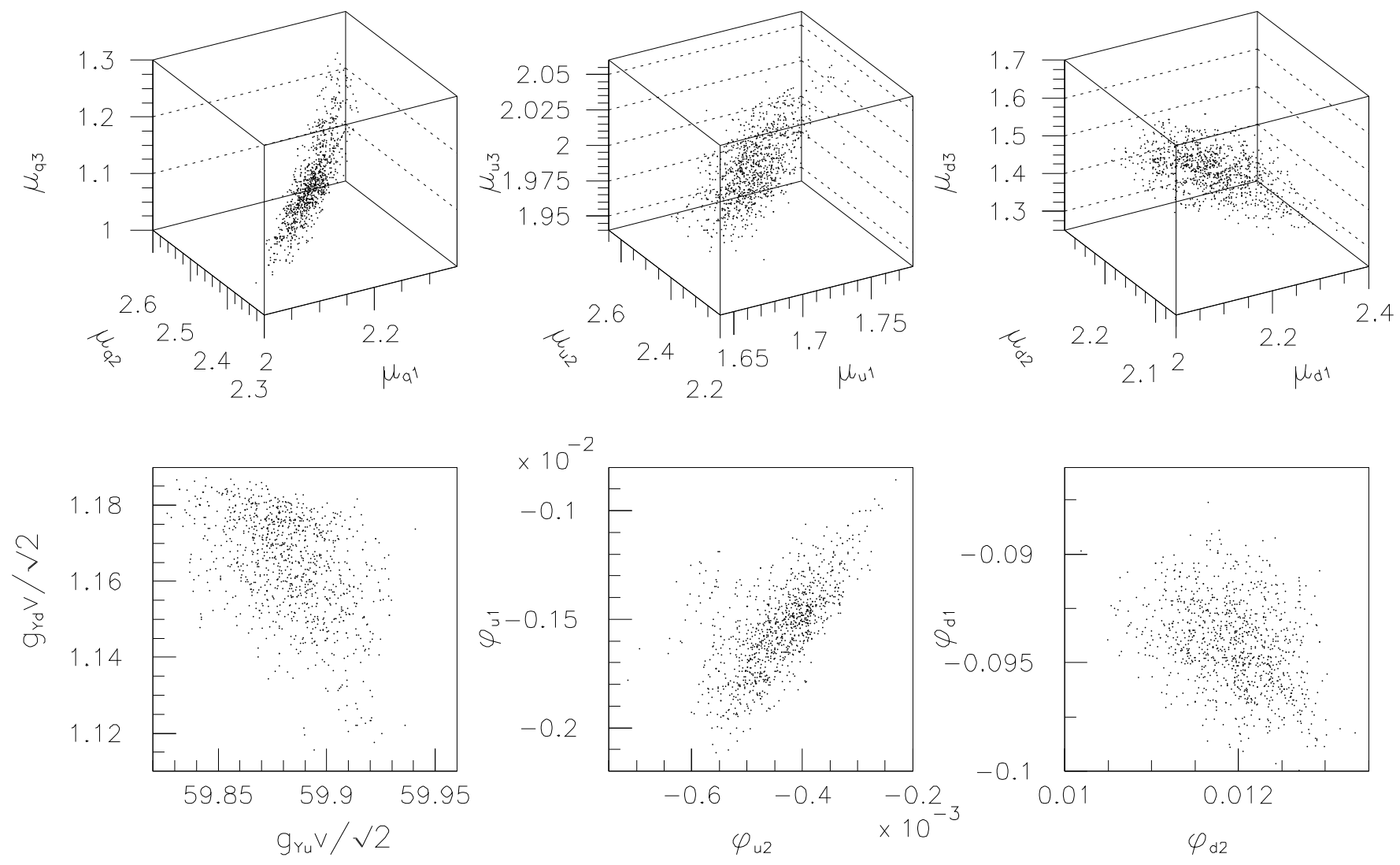

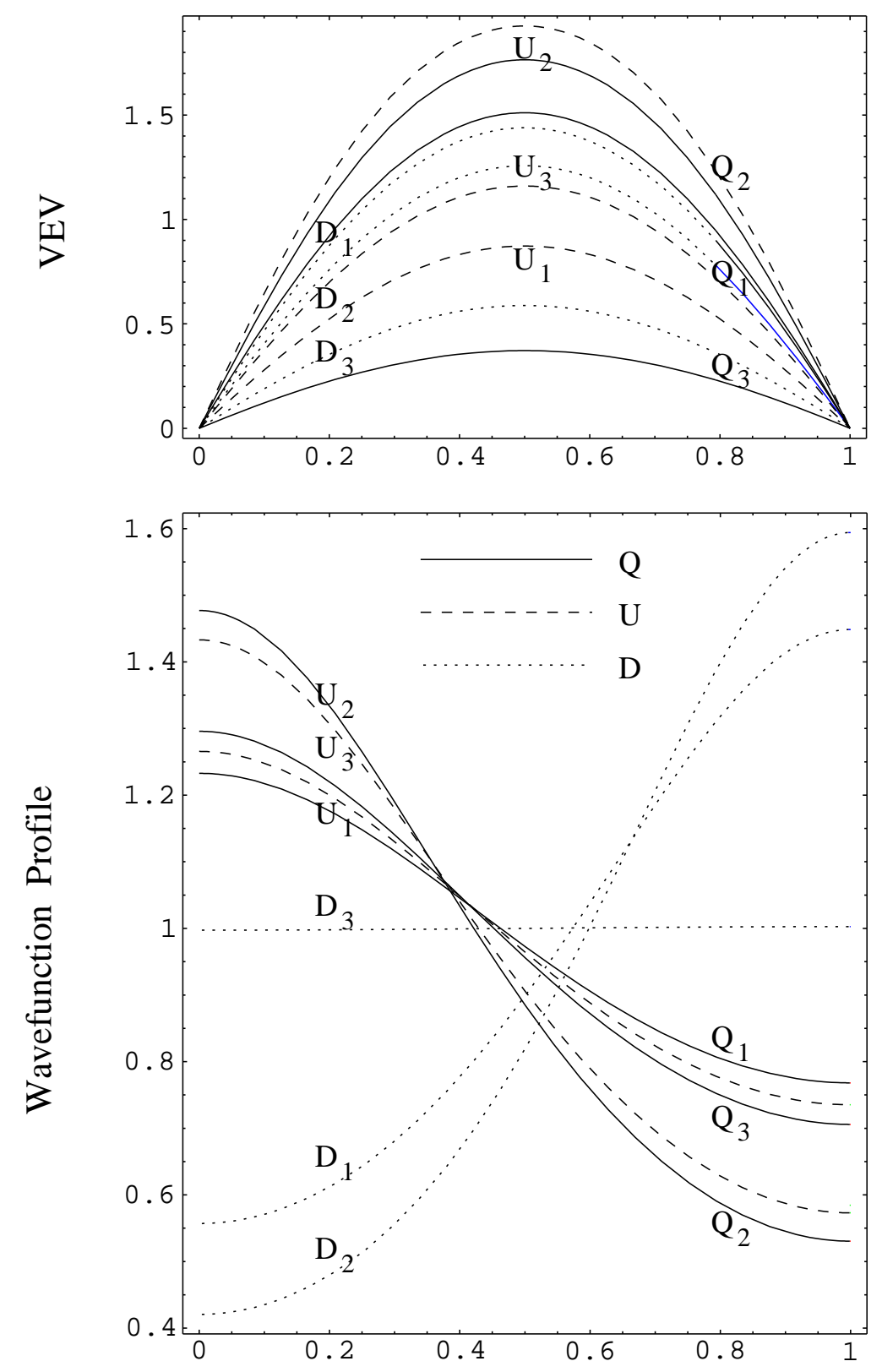

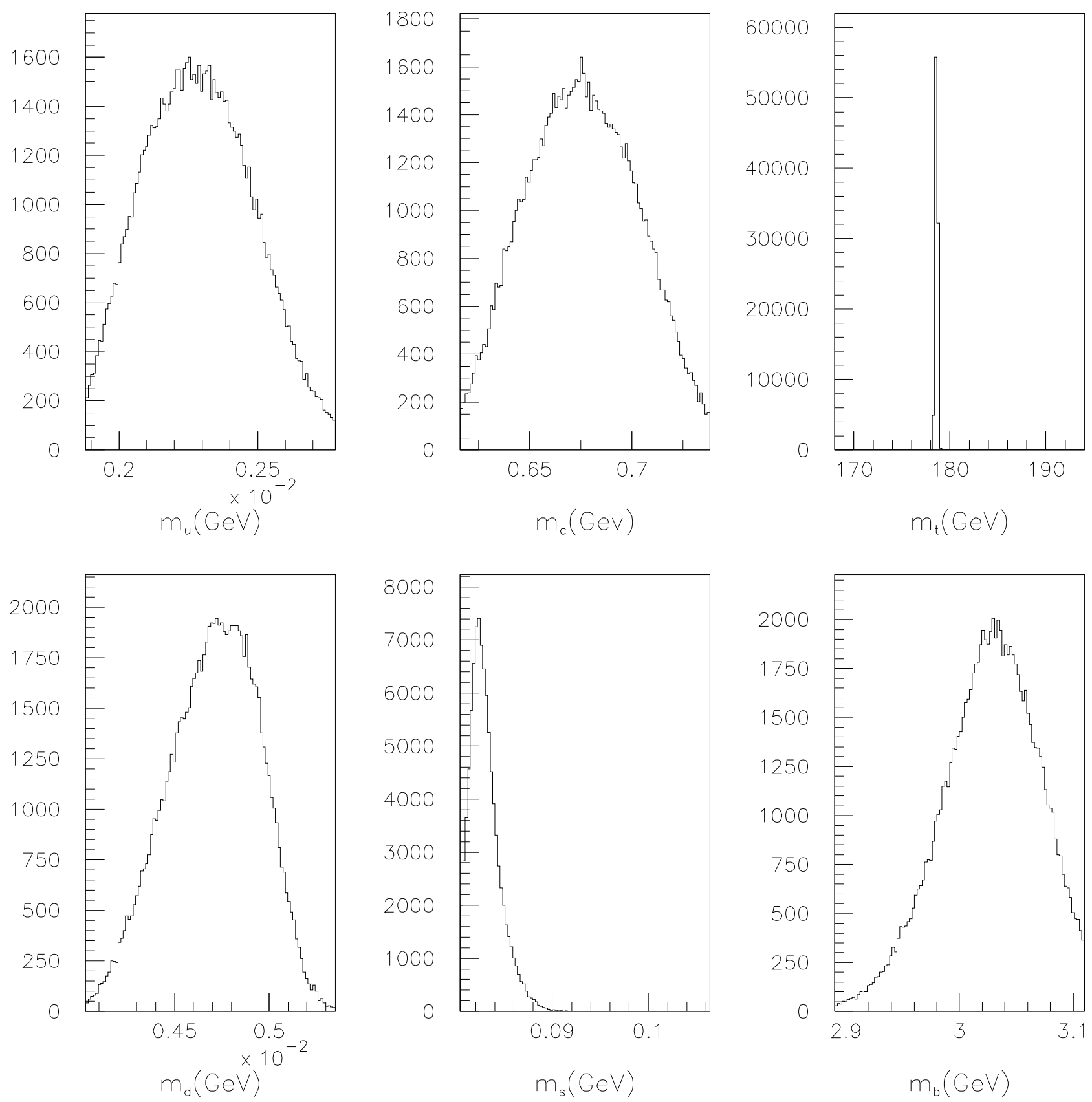

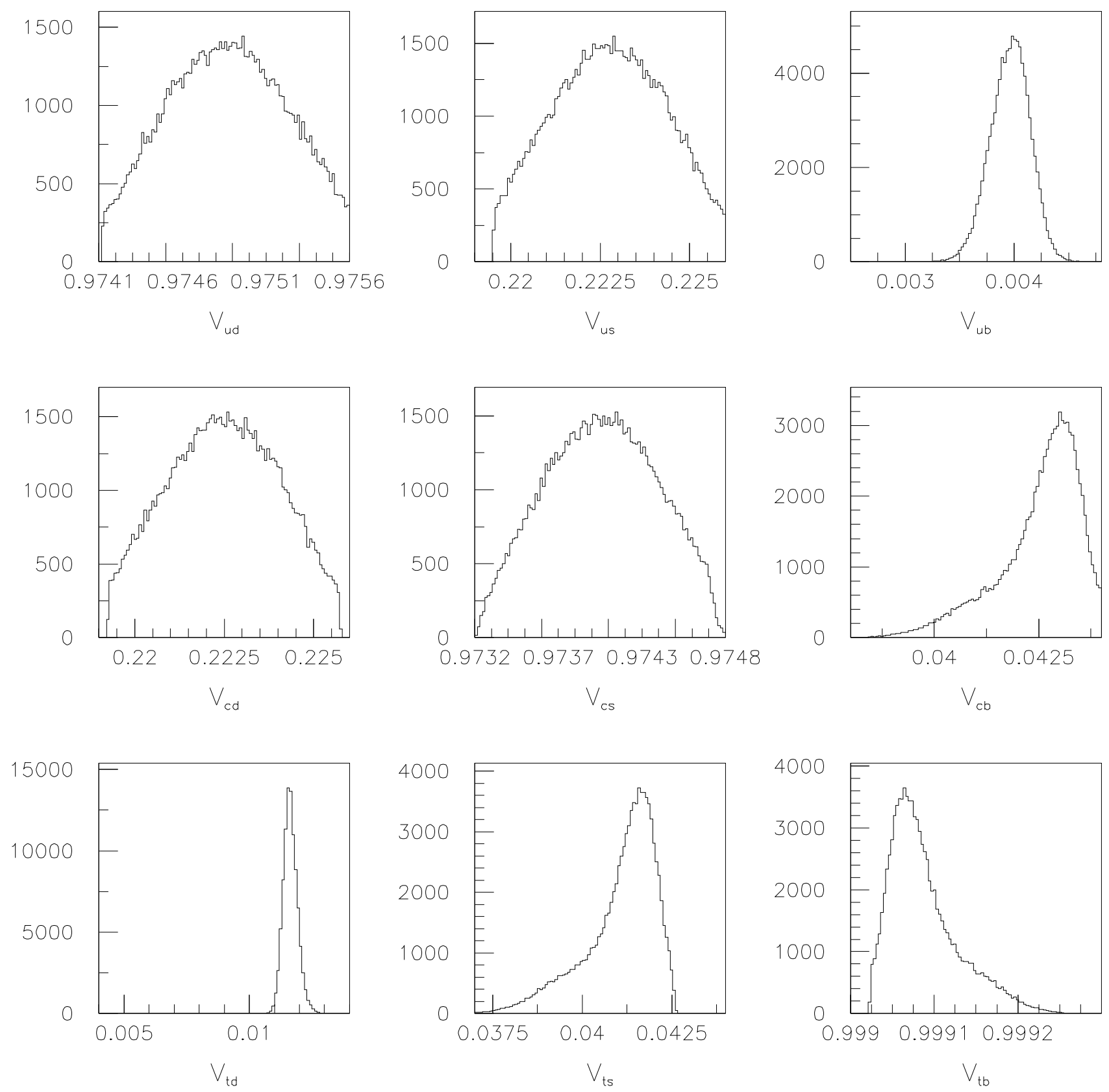


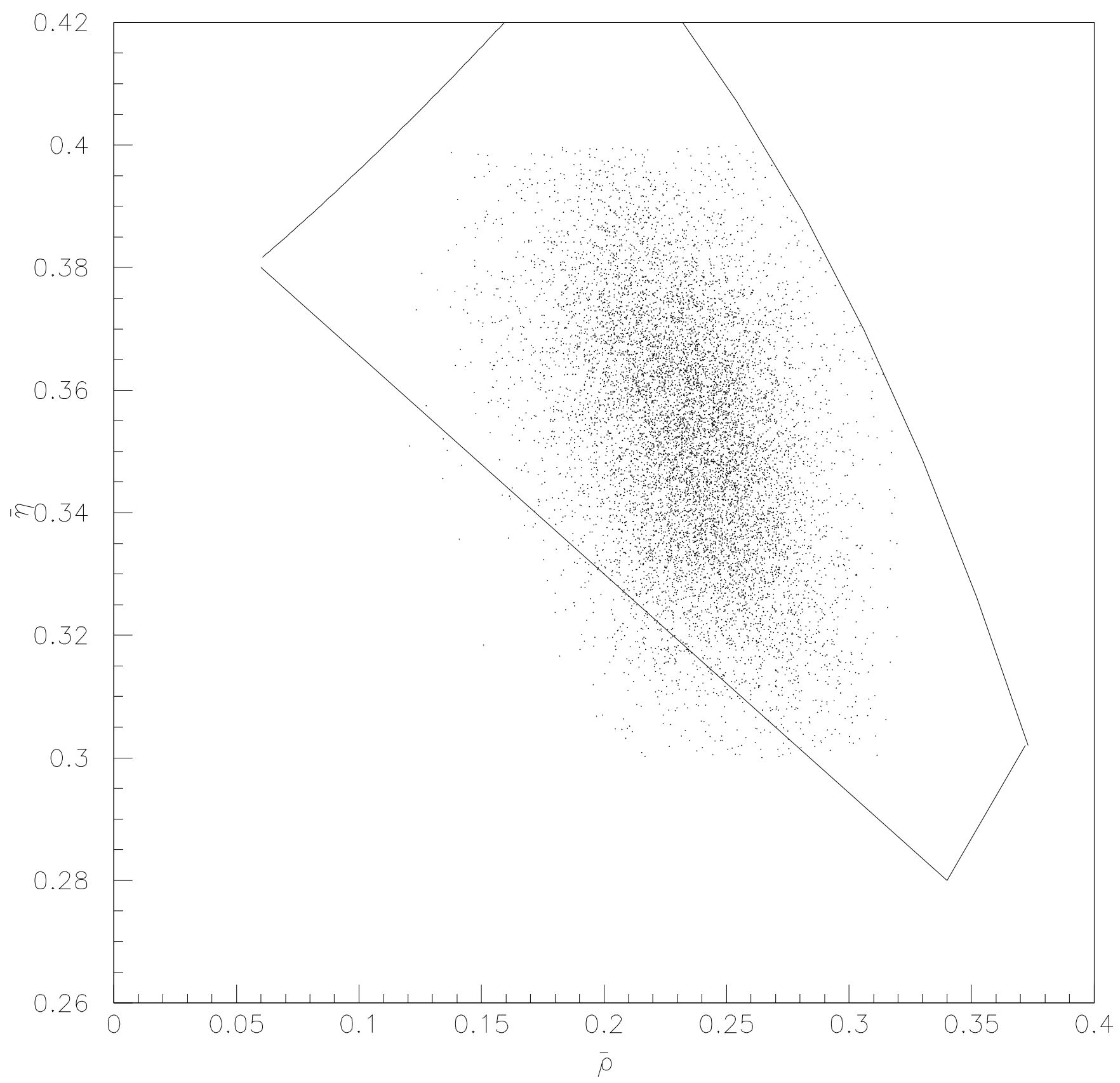



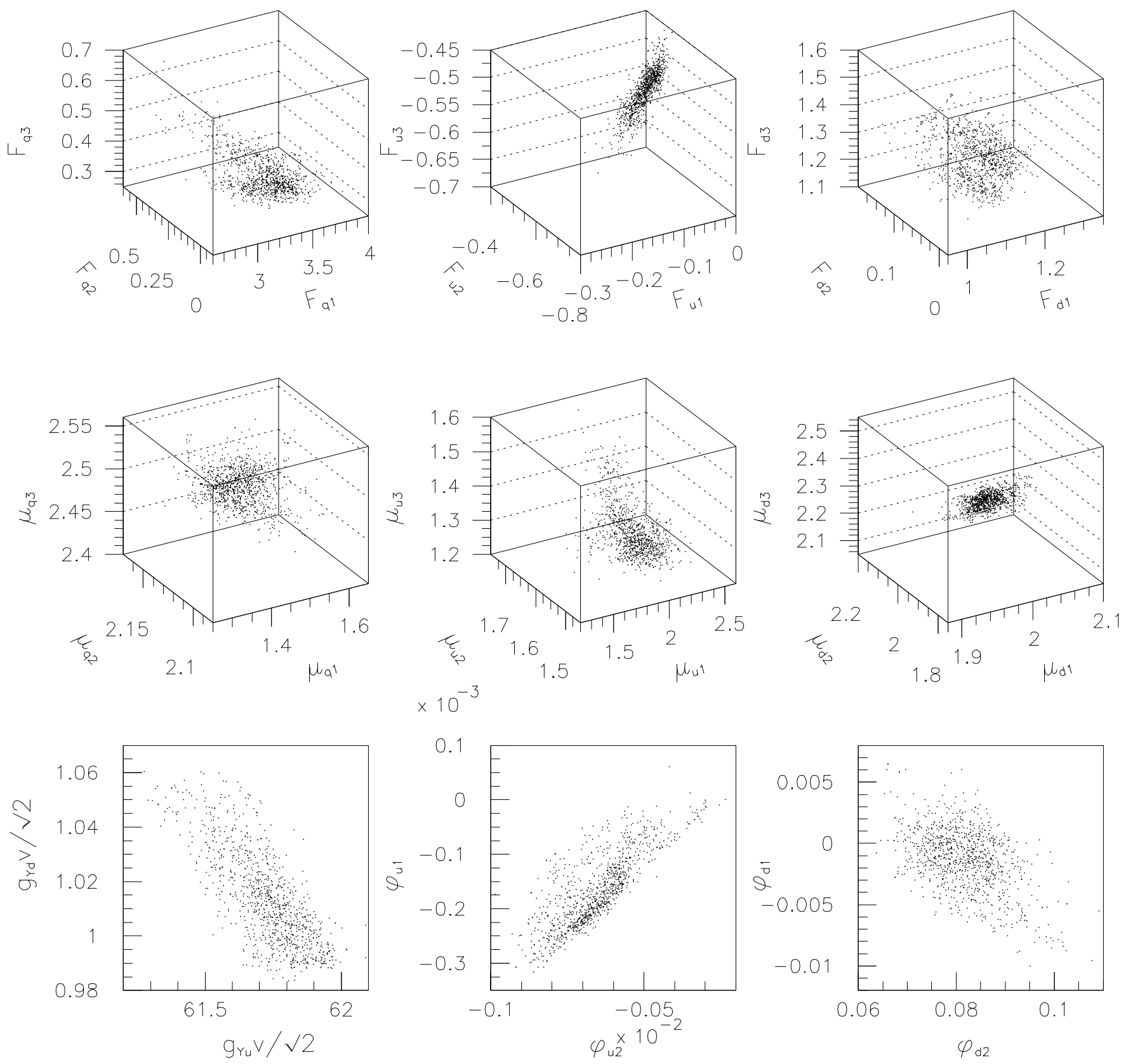

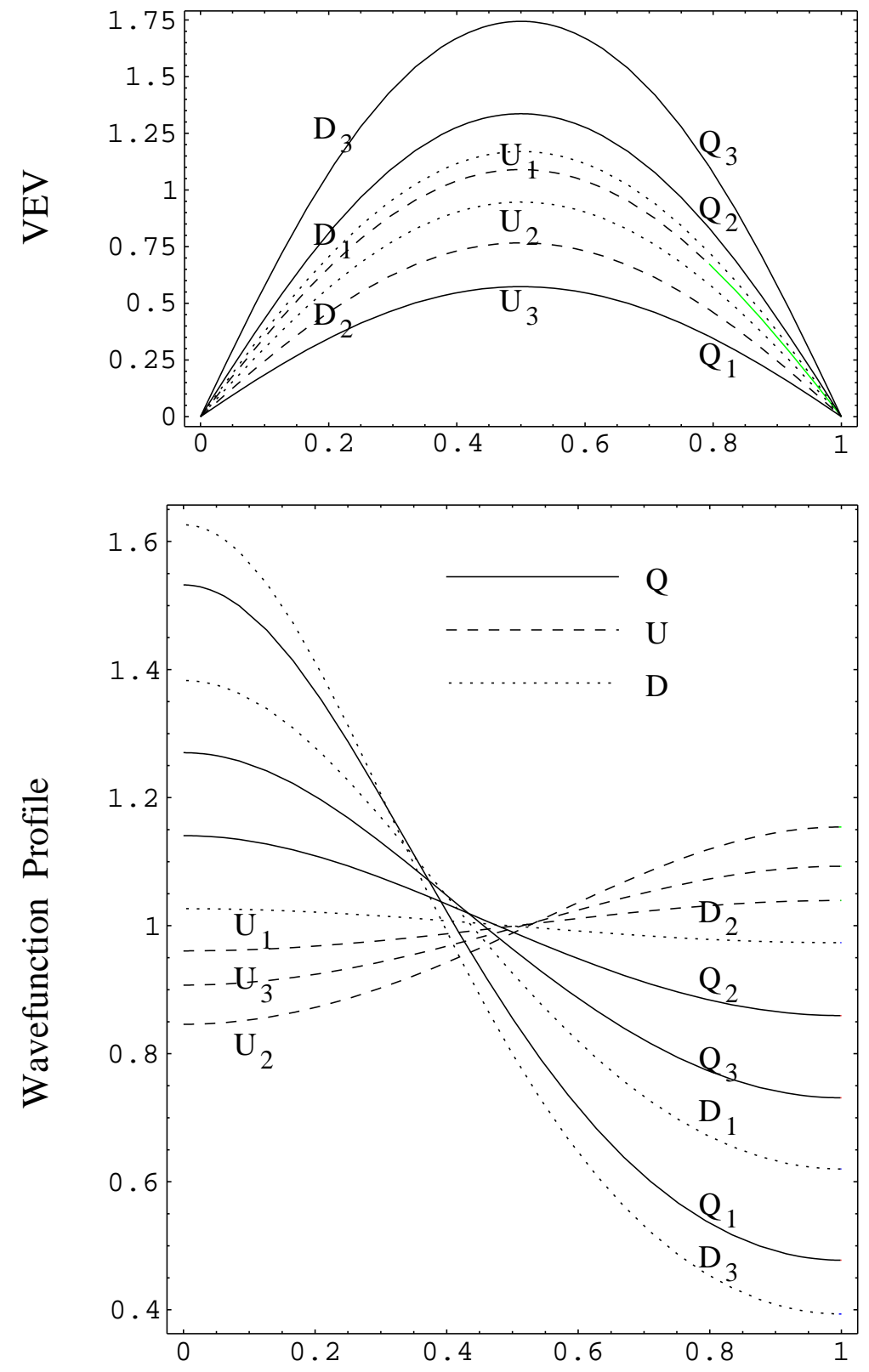

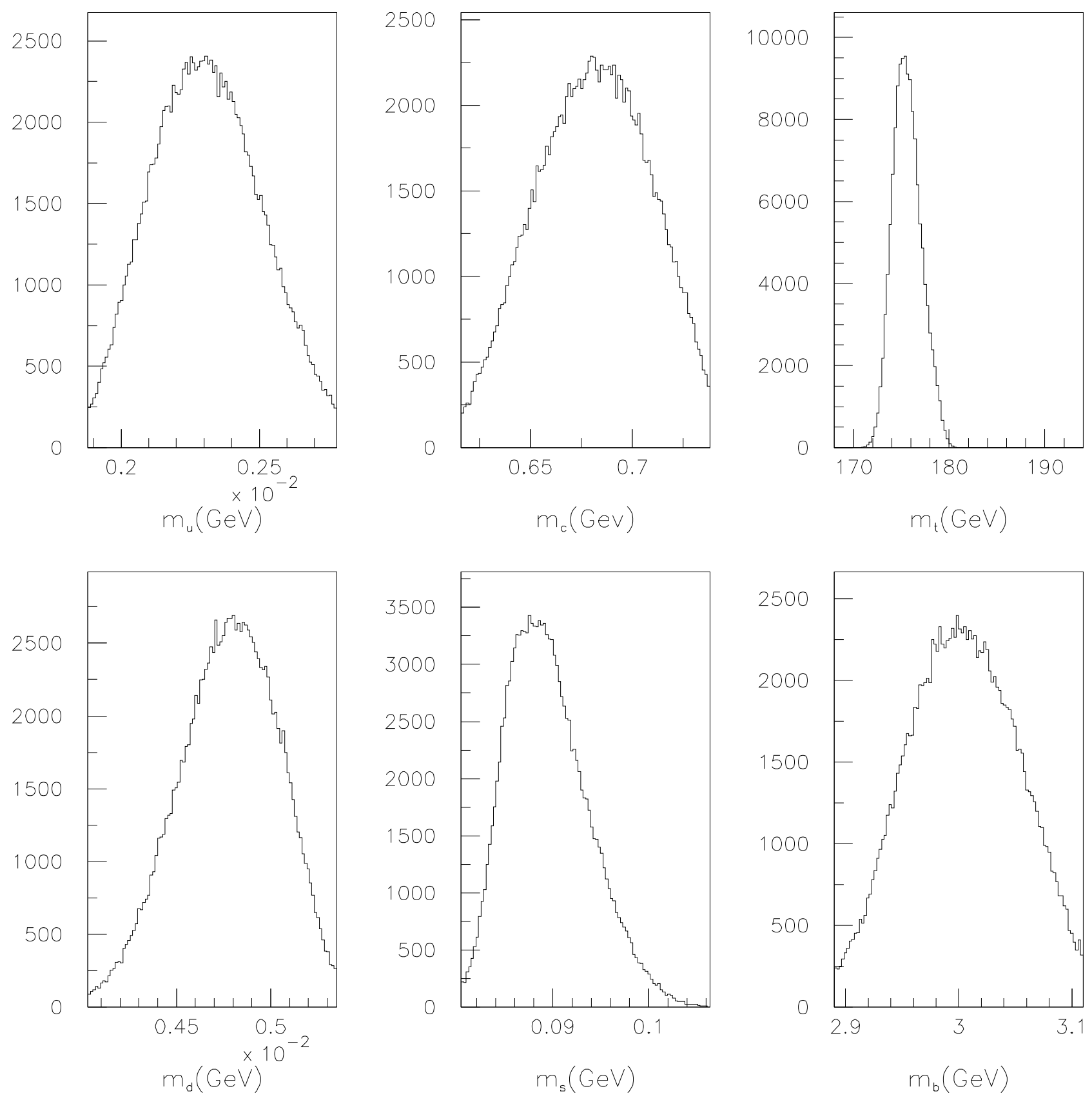

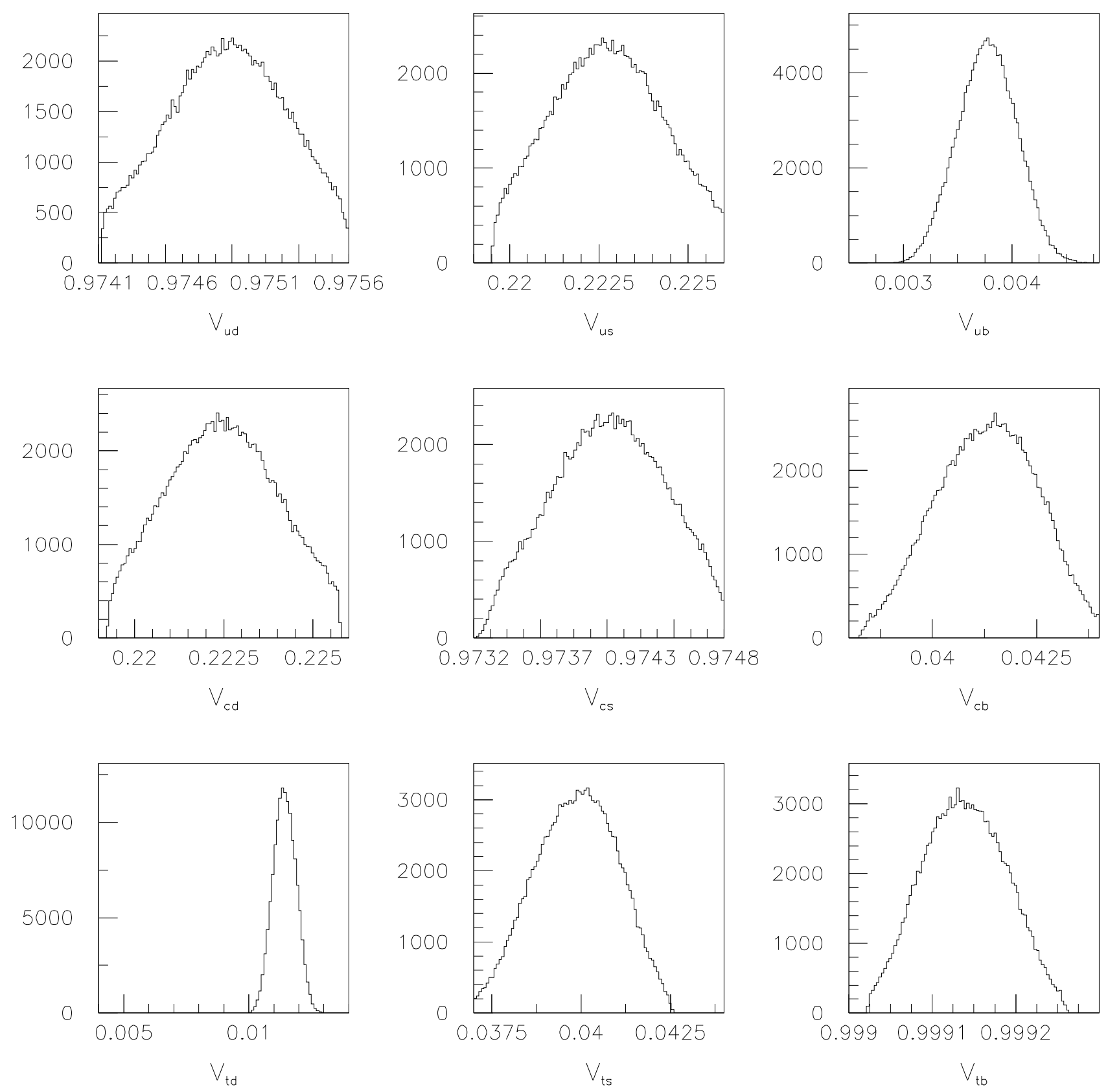


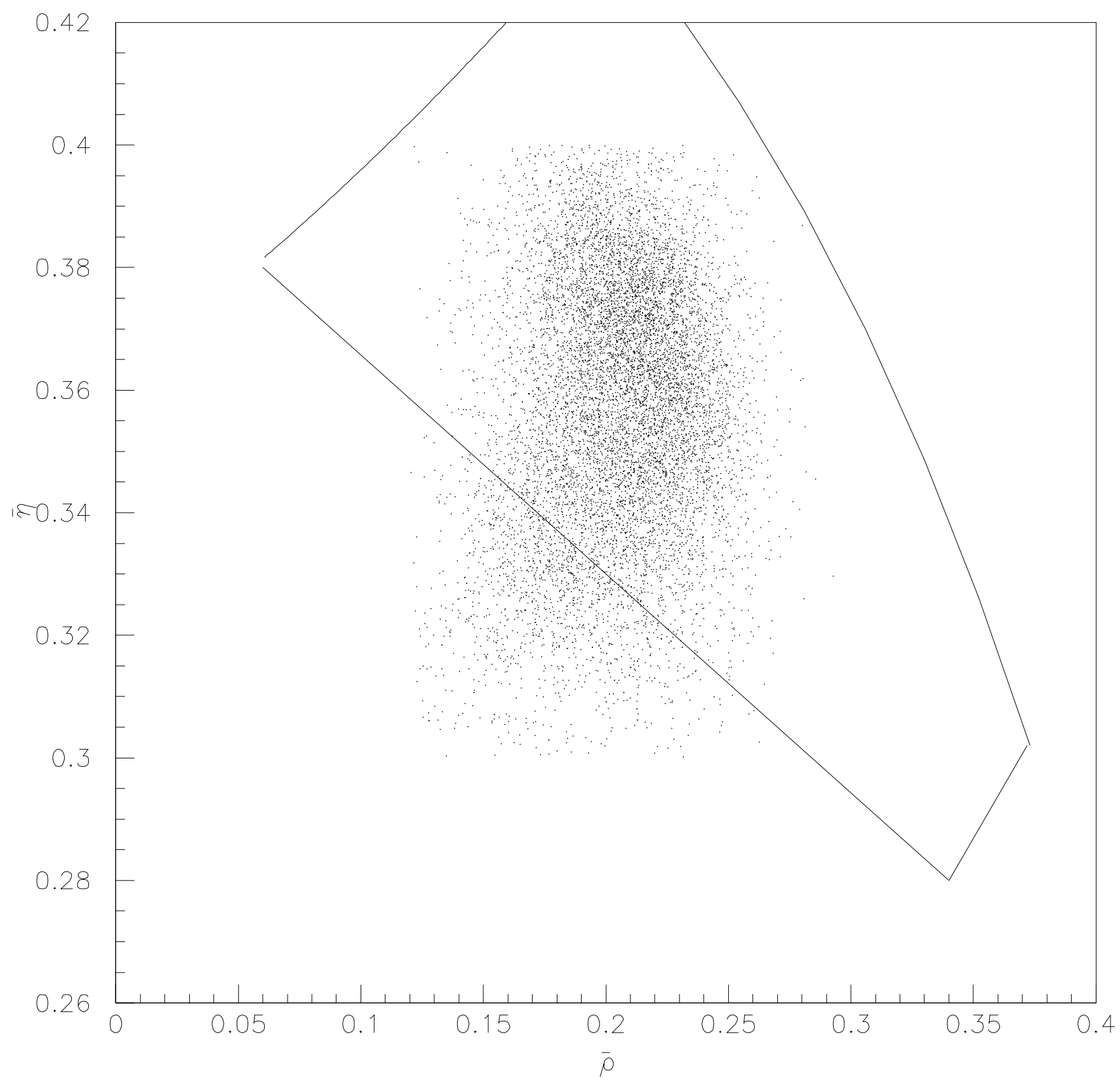



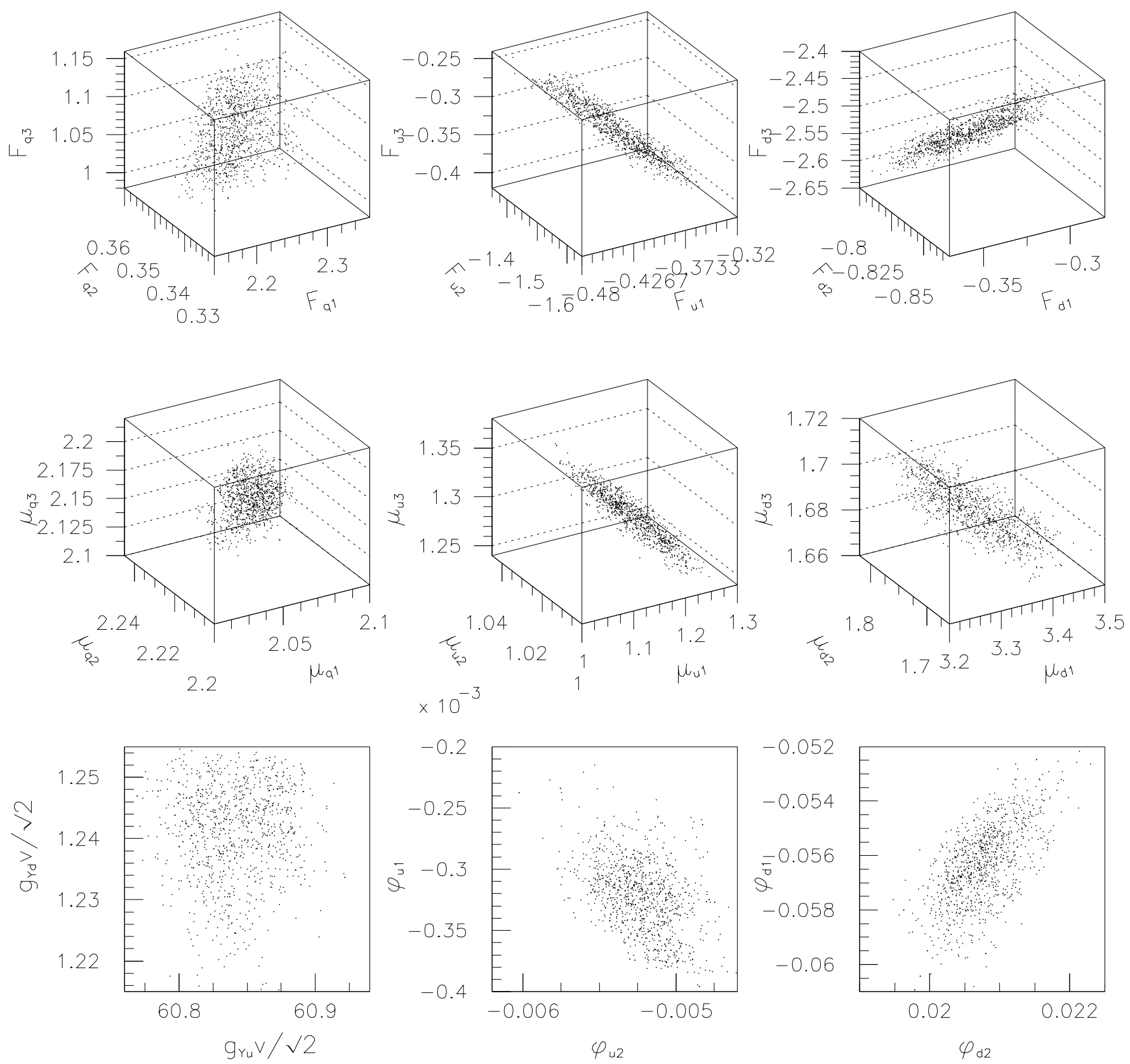

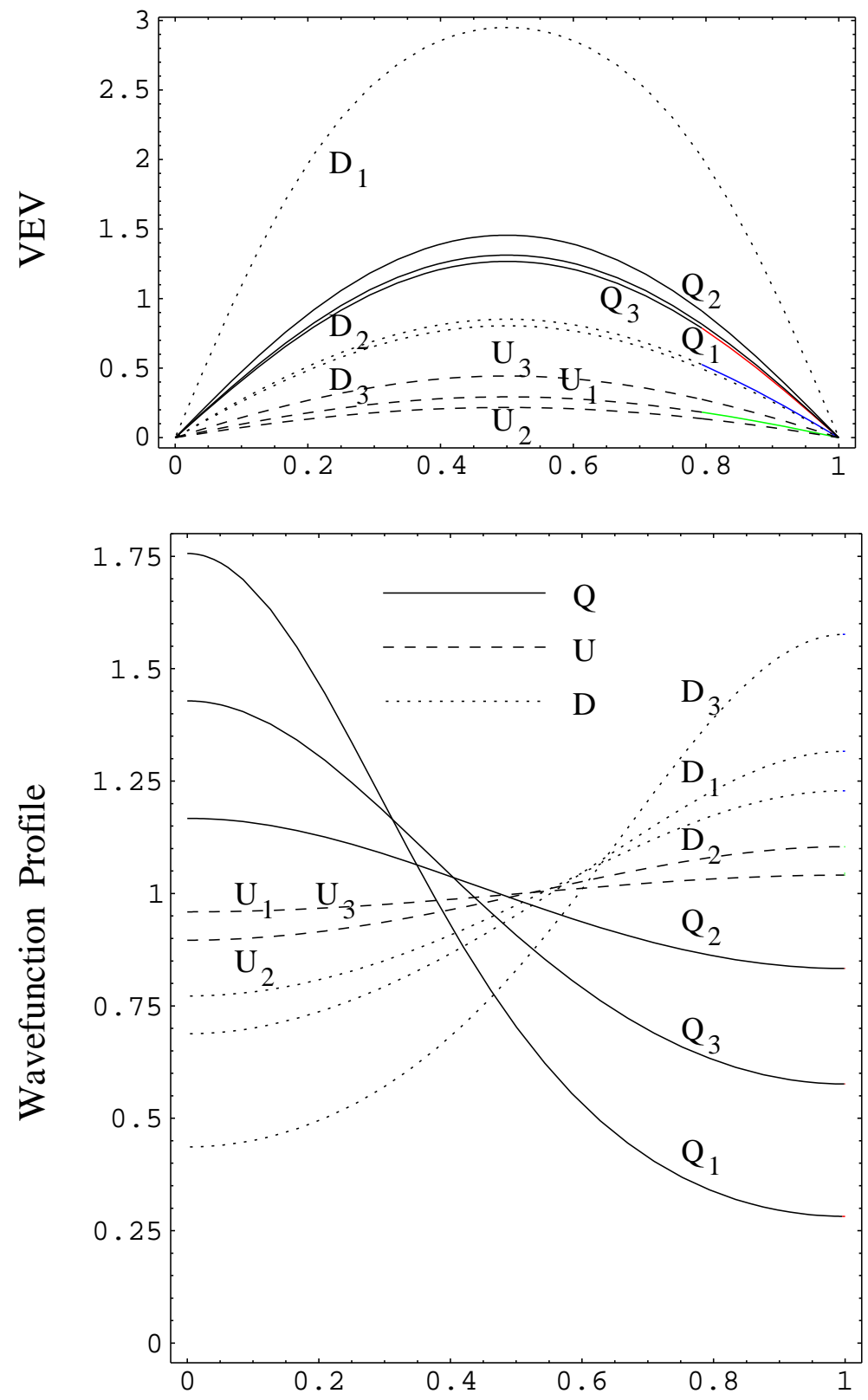

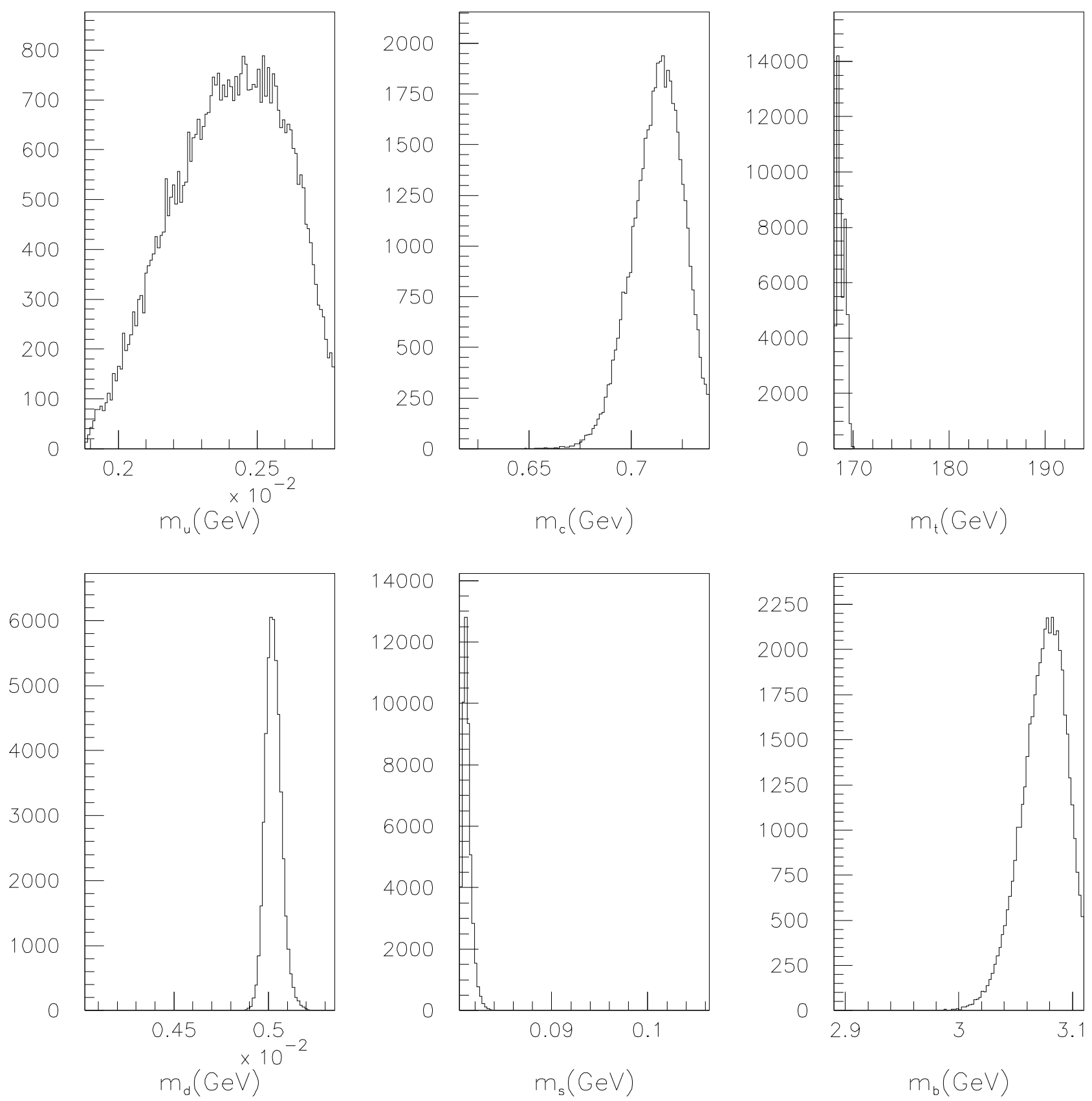

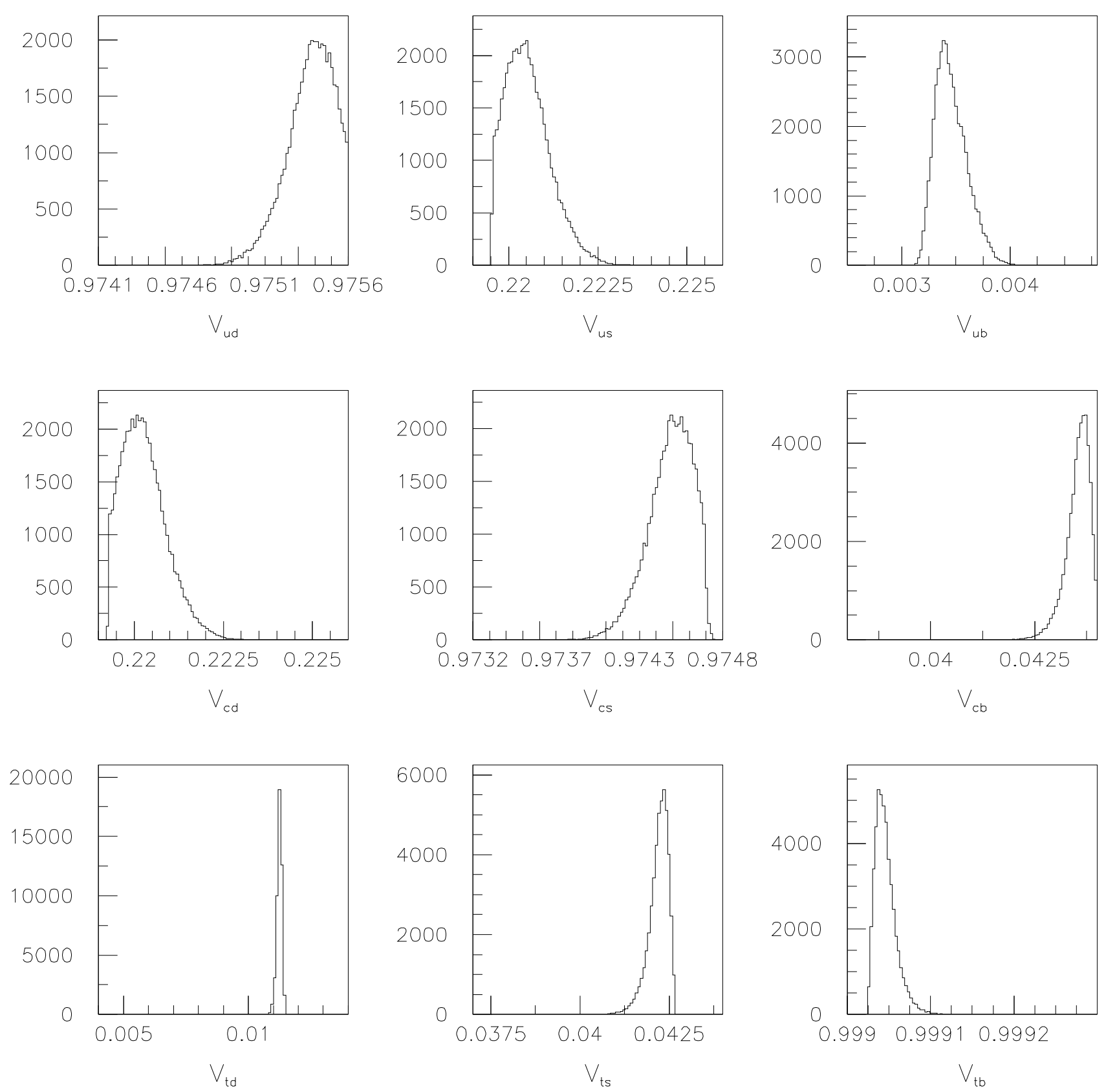


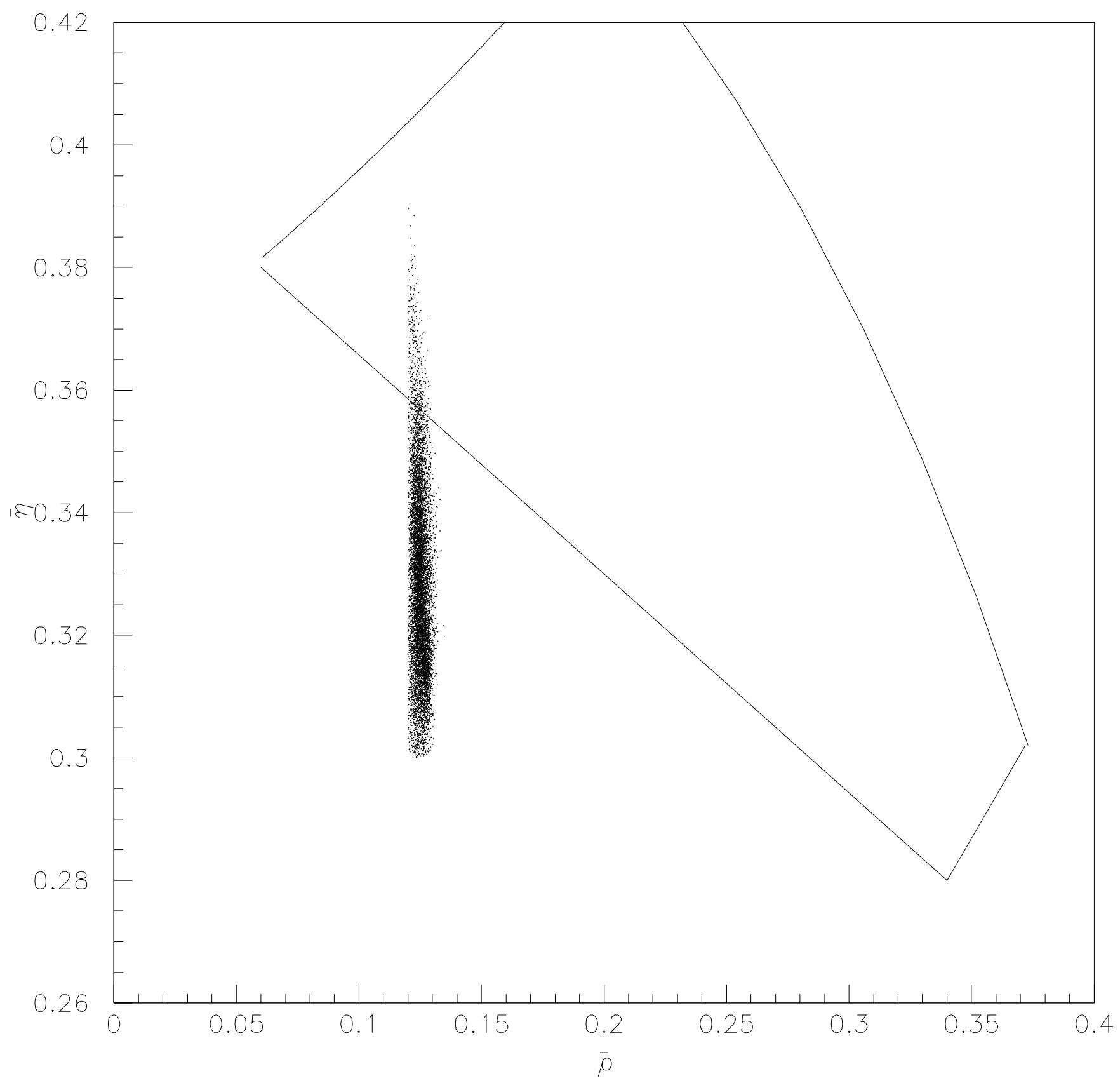



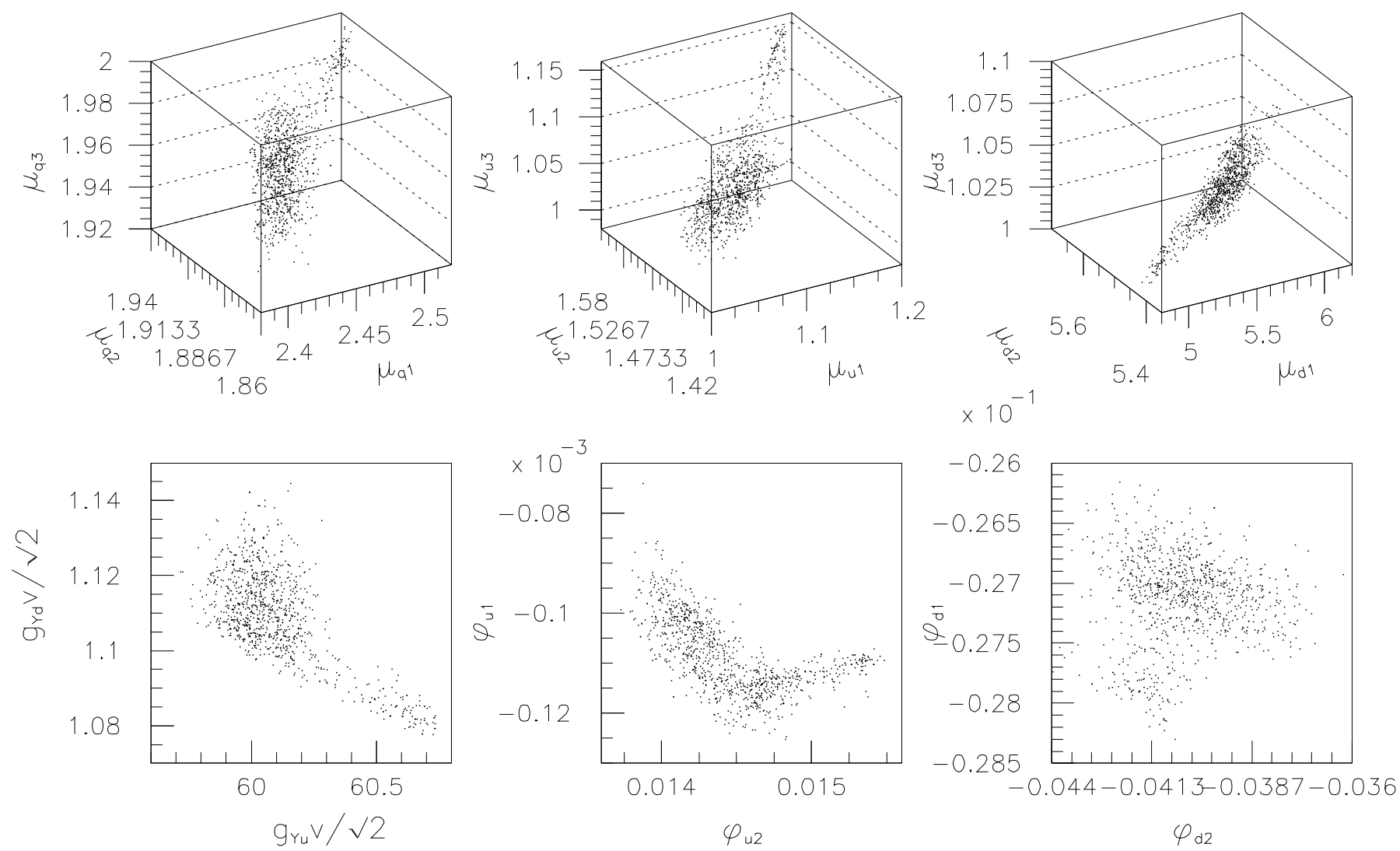

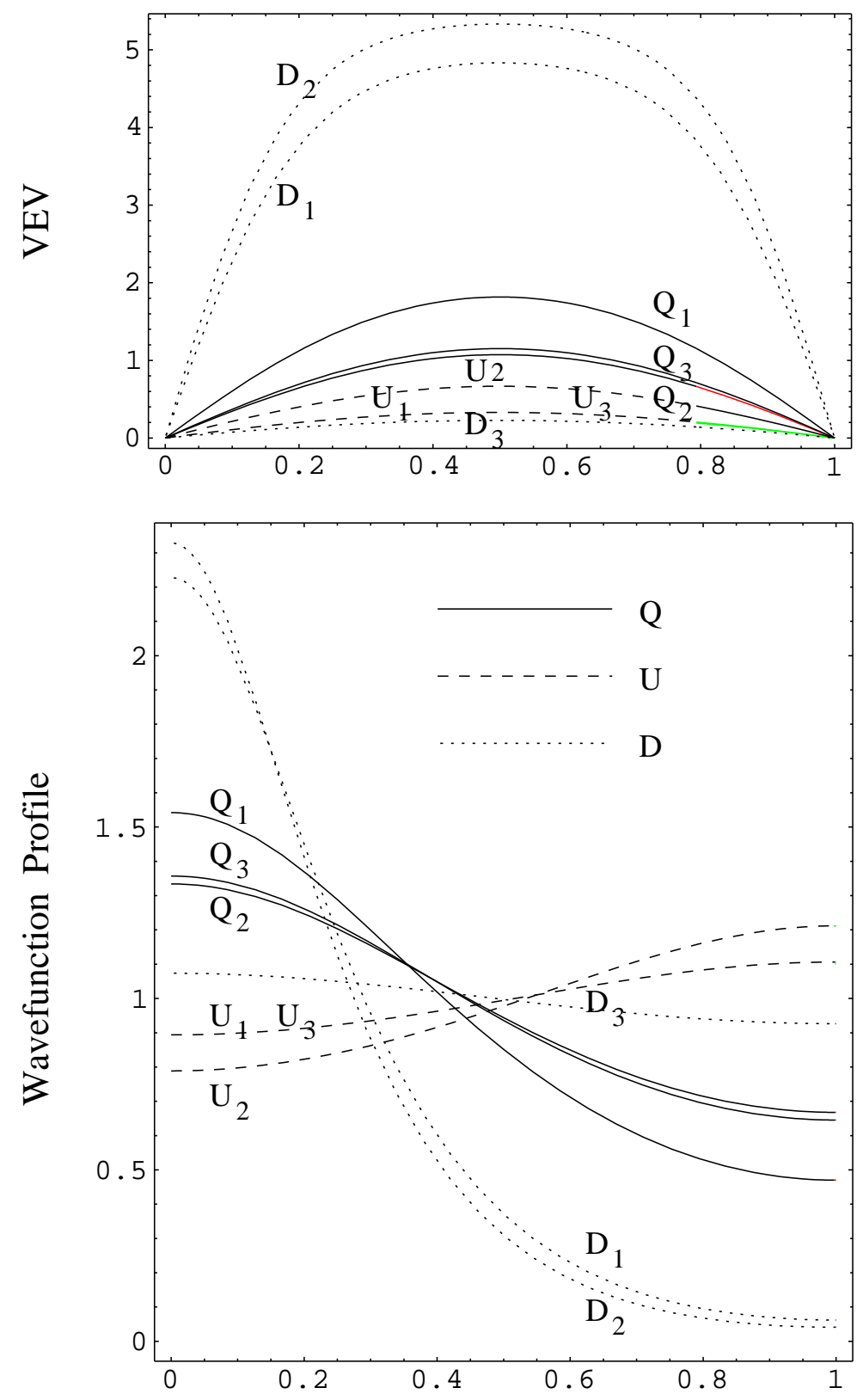

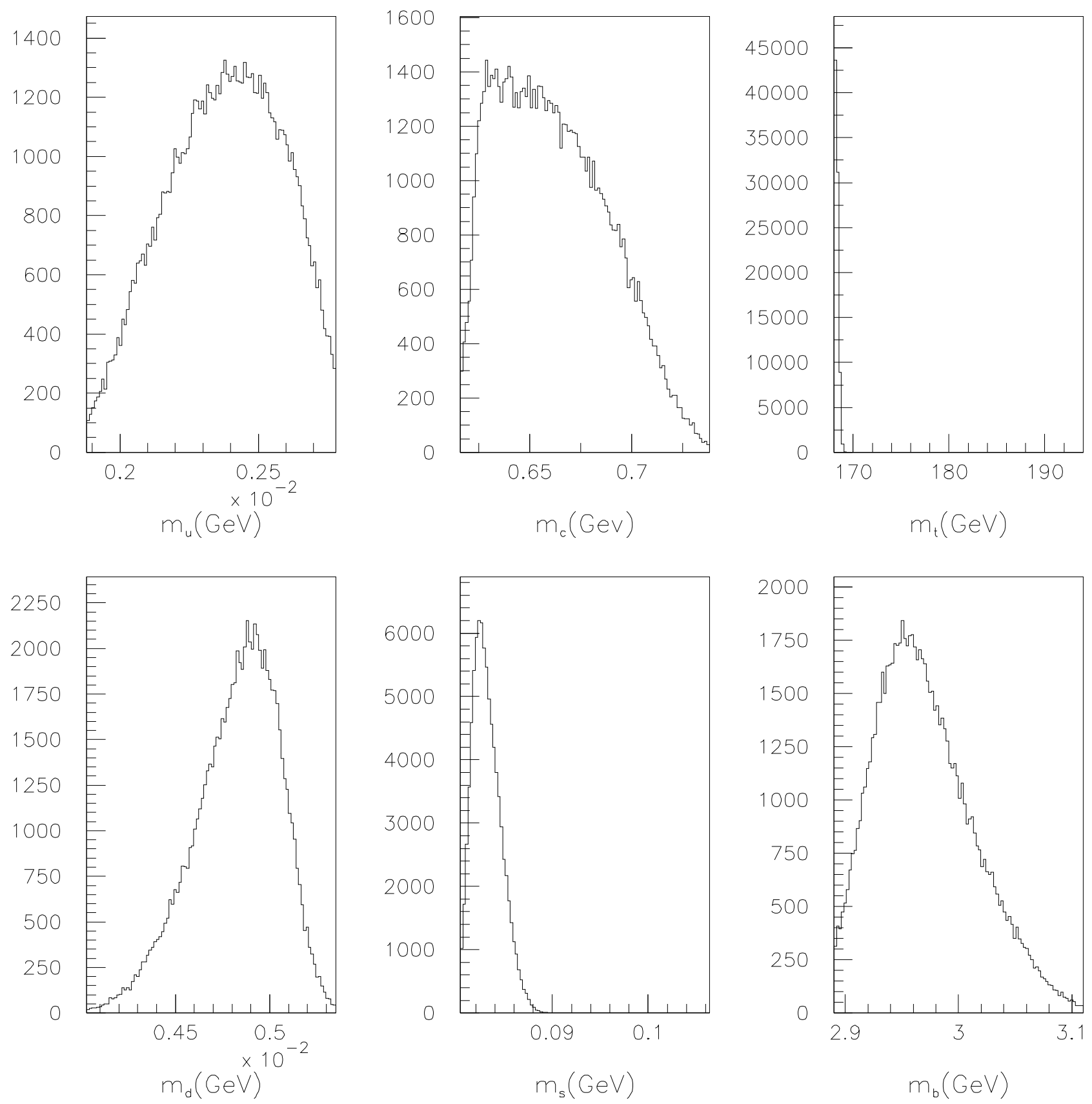

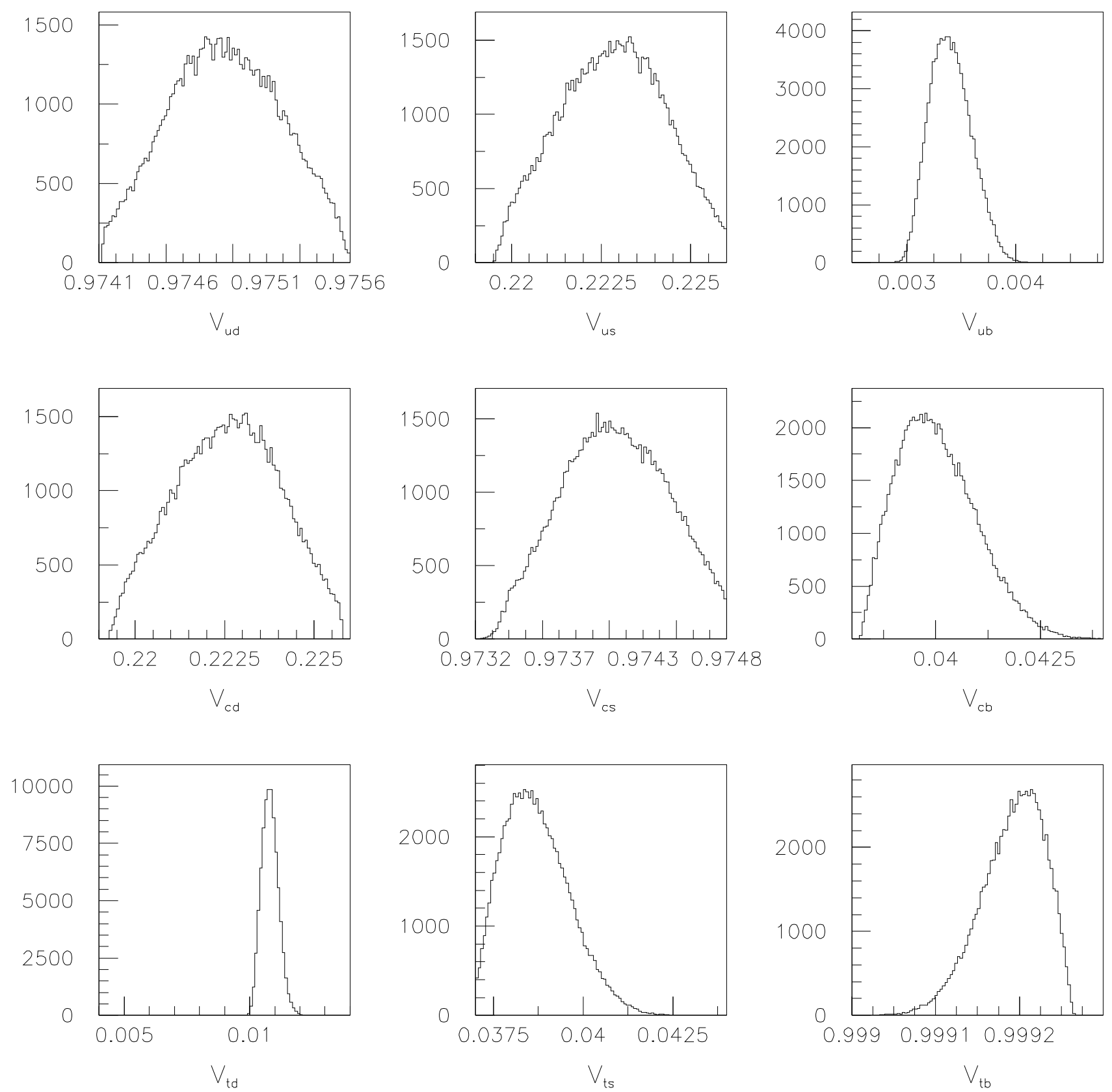


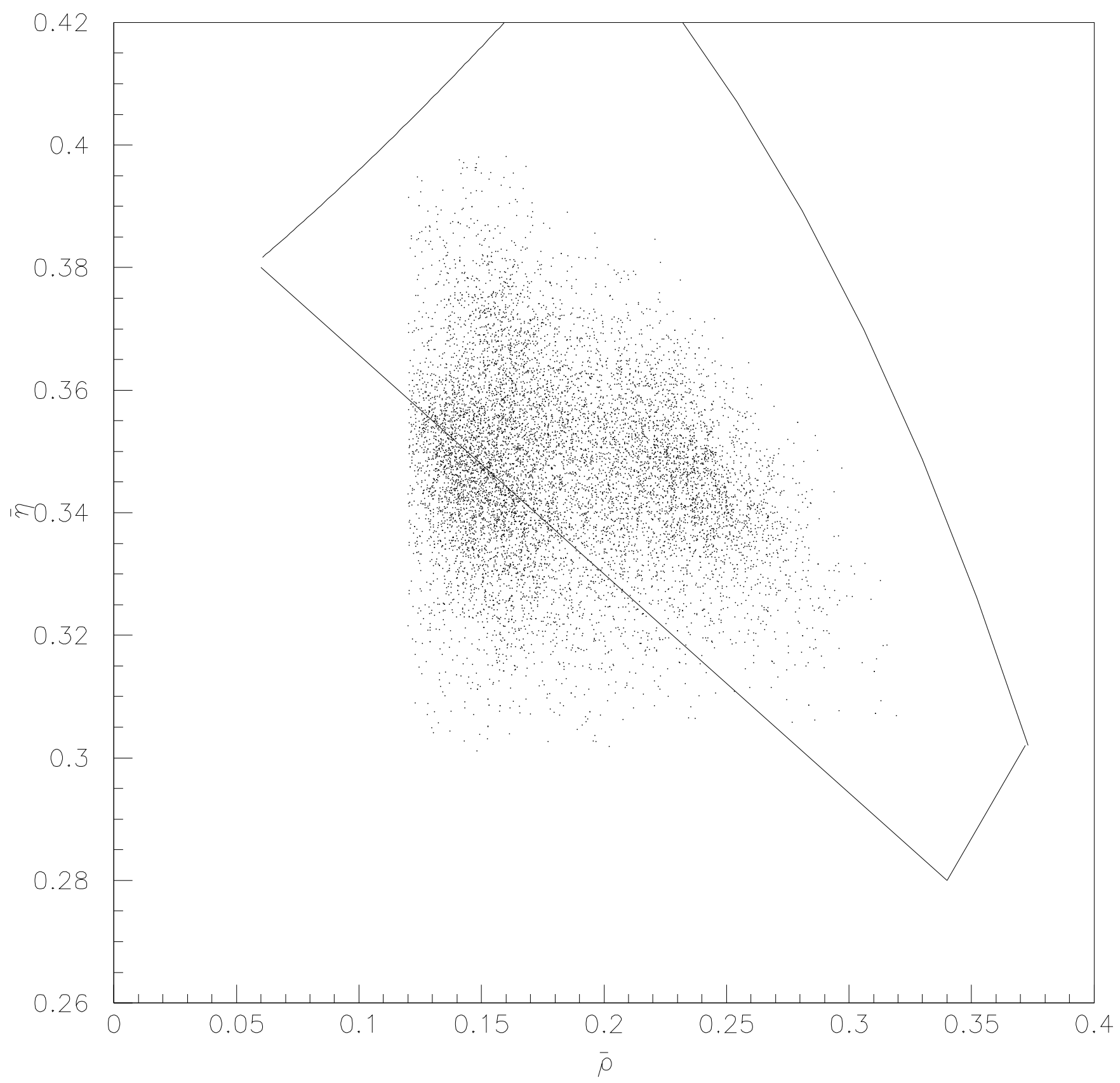

